\title{
Proximity Effects in Ferromagnet/Superconductor Heterostructures
}

\author{
K. B. Efetov ${ }^{1,2}$, I. A. Garifullin ${ }^{3}$, A. F. Volkov ${ }^{1,4}$ and K. Westerholt ${ }^{5}$ \\ 1 Institut für Theoretische Physik III, Ruhr-Universität Bochum D-44780 \\ Bochum, Germany efetov@tp3.rub.de \\ 2 L. D. Landau Institute for Theoretical Physics RAS, 119334 Moscow, Russia \\ 3 Zavoisky Physical-Technical Institute RAS, 420029 Kazan, Russia \\ ilgiz_garifullin@yahoo.com \\ 4 Institute for Radioengineering and Electronics RAS, 125009 Moscow, Russia \\ volkov@tp3.rub.de \\ 5 Institut für Experimentalphysik/Festkörperphysik, Ruhr-Universität Bochum \\ D-44780 Bochum, Germany kurt.westerholt@rub.de
}

\begin{abstract}
We review the present status of the experimental and theoretical research on the proximity effect in heterostructures composed of superconducting (S) and ferromagnetic $(\mathrm{F})$ thin films. First, we discuss traditional effects originating from the oscillatory behavior of the superconducting pair wave function in the F-layer. Then, we concentrate on recent theoretical predictions for S/F layer systems. These are a) generation of odd triplet superconductivity in the F-layer and b) ferromagnetism induced in the S-layer below the superconducting transition temperature $T_{c}$ (inverse proximity effect). The second part of the review is devoted to discussion of experiments relevant to the theoretical predictions of the first part. In particular, we present results of measurements of the critical temperature $T_{c}$ as a function of the thickness of F-layers and we review experiments indicating existence of the odd triplet superconductivity, cryptoferromagnetism and inverse proximity effect.
\end{abstract}




\section{Introduction}

If a superconducting layer $\mathrm{S}$ is brought into contact with a non superconducting metallic layer $\mathrm{N}$, the superconducting critical temperature $T_{c}$ of $\mathrm{S}$ decreases with increasing the thickness of the N-layer and the superconducting condensate penetrates into the N-layer over a long distance. This phenomenon, the conventional proximity effect, has been studied for many years starting from the beginning of the 1960's (see reviews [1, 2]).

Although attractive electron-electron interaction may be absent in the N-layer, the condensate wave function (or the Cooper pair wave function) $f\left(t-t^{\prime}\right)$ penetrates into $\mathrm{N}$ over a distance $\xi_{N}$, much exceeding the interatomic spacing. In a diffusive metal, i.e. in a metal with a high impurity concentration $\left(\tau T / \hbar<<1\right.$, where $\tau$ is the momentum relaxation time), the length $\xi_{N}$, called the correlation length, is given by $\xi_{N}=\sqrt{D / 2 \pi T}$, where $D=v_{F} l / 3$ is the diffusion coefficient, $v_{F}$ is the Fermi velocity and $l=v_{F} \tau$ is the mean free path of the conduction electrons. Magnetic impurities or a magnetic field significantly reduce the length $\xi_{N}$.

An impressive manifestation of the induced superconductivity in a normal metal is the Josephson effect in $\mathrm{S} / \mathrm{N} / \mathrm{S}$ junctions. If the thickness of the $\mathrm{N}$ layer $L$ is of the order of the correlation length $\xi_{N}$, the critical current $j_{c}$ decays exponentially with $L$ as $j_{c} \sim \exp \left(-L / \xi_{N}\right)$, which means that the characteristic length of the decay is $\xi_{N}$ and not interatomic distances. Due to this effect the Josephson critical current can still be observed even if the thickness of the N-layer exceeds $1 \mu \mathrm{m}$.

Replacing the normal metallic layer $\mathrm{N}$ in an $\mathrm{S} / \mathrm{N}$ proximity effect structure by a metallic ferromagnetic layer $\mathrm{F}$, one comes to basically the same effect: The pair wave function from $\mathrm{S}$ penetrates into $\mathrm{F}$ and makes the $\mathrm{F}$-layer superconducting. However, there are important differences, rendering the S/F proximity an interesting subject on its own.

The first important difference is that the penetration depth of the pair wave function into the F-layer is drastically reduced as compared to the $\mathrm{N}$ layer. As will be explained in the theoretical sections below, in the diffusive limit the penetration depth into the ferromagnet is given by the correlation length $\xi_{F}=\sqrt{D_{F} / 2 h}$ with the diffusion coefficient of the ferromagnet $D_{F}$ and the exchange field in the ferromagnet $h$. For strong ferromagnets like Fe, Co and $\mathrm{Ni}$, the length $\xi_{F}$ has a typical value of $0.7 \mathrm{~nm}$, i.e. the superconducting pairing function decreases in $\mathrm{F}$ exponentially on a nearly atomic length scale. The basic physical reason for this is that the exchange field in the F-layer $\mathbf{h}$ tends to align the spins of a Cooper pair and this leads to a strong pair breaking effect.

However, a faster decay of the superconducting condensate in the ferromagnet is not the only difference in comparison with the normal metals. Actually, there are other novel features of the S/F proximity effect that are less obvious and they are to be the main subject of the present review. 
Since in the F-layer the spin-up and spin-down bands are split by the exchange field $\mathbf{h}$, the electrons of a Cooper pair at the Fermi energy have necessarily different $\mathbf{k}$-vectors for the up and down spins and thus the Cooper pairs acquire a finite momentum $\Delta k$. As a consequence, the condensate function in the ferromagnet oscillates in space. As described in the theoretical chapter below, this leads to oscillations of the superconducting transition temperature $T_{c}$ as a function of the F-layer thickness. For the same reason, in Josephson junctions with an $\mathrm{S} / \mathrm{F} / \mathrm{S}$ structure, where the insulating barrier of a conventional tunnel junction is replaced by a ferromagnetic layer, the condensate function may change sign when crossing the F-layer, which leads to so-called $\pi$-type coupling of the two S-layers. This effect has been predicted long ago theoretically [3] but only recently confirmed experimentally $[4,5]$.

Following the same lines it has been demonstrated that, for a superconducting film covered from both sides by ferromagnetic layers $(\mathrm{F} / \mathrm{S} / \mathrm{F}$ - trilayer structure), the critical temperature $T_{c}$ depends on the mutual orientation of the ferromagnetic layers $[6,7]$. This is the so-called superconducting spin valve effect, the latter term originating from the possibility to switch the resistivity between the zero and a finite values by changing the mutual magnetization direction of the two ferromagnetic films. First experiments demonstrating this effect will be reviewed in the subsequent experimental section.

A fascinating new aspect of $\mathrm{S} / \mathrm{F}$ structures discovered recently is a possibility of generation of a new unconventional superconducting pairing state. The original Bardeen-Cooper-Schrieffer (BCS) theory [8] leads to a conventional s-wave pairing and for several decades this type of superconductivity has been remaining the only one observed experimentally. On the other hand, the superconductivity in high- $T_{c}$ cuprates discovered later shows a d-wave symmetry or a mixture of s- and d-wave components of the order parameter [9]. Both the s-wave and d-wave types of the symmetries of the order parameter usually imply the singlet pairing, which means that the total spin of the Cooper pair is zero. In this case, the order parameter $\Delta_{\alpha \beta}$ has the form $\Delta_{\alpha \beta}=\Delta(\mathbf{k}) \cdot\left(i \sigma_{2}\right)_{\alpha \beta}$, where $\sigma_{2}$ is the second Pauli matrix in the spin space and $\Delta(\mathbf{k})$ is a function of the momentum $\mathbf{k}$. As the spin part is antisymmetric with respect to transposition of the spin indices, the antisymmetricity of the order parameter following from the Pauli principle is fulfilled provided the function $\Delta(\mathbf{k})$ is even $(\Delta(\mathbf{k})=\Delta(-\mathbf{k}))$.

Another type of pairing, the spin-triplet superconductivity, has been discovered in materials with strong electronic correlations, namely, in heavy fermion intermetallic compounds as well as in organic materials (for a review see [10]). Recently, a lot of work has been devoted to studying superconducting properties of $\mathrm{Sr}_{2} \mathrm{RuO}_{4}$ and convincing experimental data in favor for the triplet p-wave superconductivity have been obtained. We refer the reader to the review articles by [11] and [12].

In contrast to the singlet superconductivity, the spin part of the order parameter for the triplet superconductivity is symmetric with respect to exchanging the spin indices. Assuming that the order parameter (or the conden- 
sate function) does not depend on frequency (which is a standard assumption) one comes to the conclusion that the order parameter must be antisymmetric with respect to the inversion of the momentum or, equivalently, to transposition of the coordinates. This is this type of the superconductivity that has been observed in, e.g., heavy fermions.

Still, there is one more, a very non-trivial possibility for triplet pairing first predicted in Ref. [42] that may be realized in S/F systems. It turns out that the triplet pairing is also possible when the condensate is an even function of momentum and an odd function of the Matsubara frequency. As will be described in the theoretical sections below, a corresponding component of the condensate function can be generated at the S/F interface by an inhomogeneous in space magnetization. First experimental evidence in favor of the existence of such an odd triplet superconductivity has been reported recently $[13,14,15]$. A hallmark of the triplet superconductivity in the $\mathrm{S} / \mathrm{F}$ systems is its large penetration depth in the F-layer, which follows from the fact that the exchange field in the ferromagnet does not break the triplet pairs. Moreover, in contrast to the "conventional" triplet pairing, the odd triplet superconductivity is not sensitive to non-magnetic disorder and therefore is very robust.

Last but not least, in the $\mathrm{S} / \mathrm{F}$ layer systems the conventional proximity effect is not the only interesting phenomenon caused by the mutual influence of ferromagnetic and superconducting order. As will be explained below, the ferromagnetic state of the F-layer can in its turn be strongly modified by the presence of the superconductor, an effect that is usually referred to as cryptoferromagnetism $[16,17,18]$.

Remarkably, not only supercoductivity can penetrate ferromagnets but also the S-layers can become ferromagnetic [19]. For the latter phenomenon the term inverse proximity effect was coined.

Thus, one can see that the proximity effect not only exists in the S/F structures but there are many of them. This makes these systems extremely interesting for both theorists and experimentalists. In the following sections we analyze the proximity effects in the $\mathrm{S} / \mathrm{F}$ systems from both theoretical and experimental points of view. Although several reviews on the proximity effects have been recently published [20, 21, 22, 23, 24, 25], we emphasize novel developments and open problems. At the same time, we focus mainly on our own recent work.

\section{Proximity effect: Theory}

\subsection{S/F structures: uniform magnetization of the ferromagnet}

In this section we review shortly the main theoretical results on what happens if one replaces the normal metal $\mathrm{N}$ in a $\mathrm{N} / \mathrm{S}$ proximity structure by a ferromagnetic metal $\mathrm{F}$. The effective ferromagnetic exchange field acts on spins of the conduction electrons in the ferromagnet resulting in an additional term 
$\hat{H}_{e x}$ for this interaction in the total Hamiltonian $\hat{H}_{t o t}$ describing the proximity effect:

$$
\hat{H}_{t o t}=\hat{H}+\hat{H}_{e x}, \quad \hat{H}_{e x}=-\int d^{3} \mathbf{r} \psi_{\alpha}^{+}(\mathbf{r})\left(\mathbf{h}(\mathbf{r}) \sigma_{\alpha \beta}\right) \psi_{\beta}(\mathbf{r}) d \mathbf{r},
$$

where $\psi^{+}(\psi)$ are creation and destruction operators, $\mathbf{h}$ is the exchange field, $\sigma_{\alpha \beta}$ are Pauli matrices, and $\alpha, \beta$ are spin indices. The operator $\hat{H}$ stands for a non-magnetic part of the Hamiltonian. It includes the kinetic energy, impurities, external potentials, etc. and is sufficient to describe all properties of the system in the absence of the exchange field $\mathbf{h}$.

The energy of spin-up electrons differs from the energy of spin-down electrons by the Zeeman energy $2 h$. All functions, including the condensate Green's function $f$, become matrices in the spin space with non-zero, diagonal and off-diagonal elements. In this subsection we consider the case of a homogeneous magnetization $M$. In this situation the matrix $\hat{f}$ is diagonal and can be represented in the form

$$
\hat{f}=f_{3} \hat{\sigma}_{3}+f_{0} \hat{\sigma}_{0},
$$

where $f_{3}$ is the amplitude of the singlet component and $f_{0}$ is the amplitude of the triplet component with zero projection of the magnetic moment of the Cooper pairs on the $\mathrm{z}$ axis $(S=0)$. Note that for $\mathrm{S} / \mathrm{N}$ structures the condensate function has a singlet structure only, i.e. it is proportional to $\hat{\sigma}_{3}$. The presence of the exchange field $\mathbf{h}$ leads to the appearance of the triplet term proportional to $\hat{\sigma}_{0}$. In general (non-homogeneous magnetization) case, the matrix $\hat{f}$ contains not only the matrices $\hat{\sigma}_{0,3}$ but also the matrices $\hat{\sigma}_{1,2}$.

The amplitudes of the singlet and triplet components are related to the correlation functions $\left\langle\psi_{\alpha} \psi_{\beta}\right\rangle$ as follows [26, 27]:

$$
f_{0}(t) \sim\left\langle\psi_{\uparrow}(t) \psi_{\downarrow}(0)\right\rangle+\left\langle\psi_{\downarrow}(t) \psi_{\uparrow}(0)\right\rangle, \quad f_{3}(t) \sim\left\langle\psi_{\uparrow}(t) \psi_{\downarrow}(0)\right\rangle-\left\langle\psi_{\downarrow}(t) \psi_{\uparrow}(0)\right\rangle .
$$

One can see that a permutation of spins does not change the function $f_{3}(0)$, whereas such a permutation leads to a change of the sign of $f_{0}(0)$. This means that the amplitude of the triplet component taken at equal times is zero in agreement with the Pauli exclusion principle. Later we will see that in the case of a non-homogeneous magnetization all triplet components including $\left\langle\psi_{\uparrow}(t) \psi_{\uparrow}(0)\right\rangle$ and $\left\langle\psi_{\downarrow}(t) \psi_{\downarrow}(0)\right\rangle$ differ from zero.

Let us begin with a discussion of properties of the F/S systems with homogeneous magnetization. The exchange interaction tends to align the spins of the free electrons in one direction whereas the superconducting correlations result in formation of Cooper pairs consisting of electrons with opposite spins. Therefore, the superconducting transition temperature $T_{c}$ of an $\mathrm{S} / \mathrm{F}$ bilayer system should be considerably reduced in S/F structures provided the interface transparency is high, i.e. if the electron can travel freely from the $\mathrm{S}$ to the F side and vice versa. However, it turns out that the dependence of $T_{c}$ on 
the exchange field $h$ and on the thickness of S- or F-layers is nontrivial: the critical temperature $T_{c}$ may vary with increasing $d_{F}$ in a non-monotonic way.

The critical temperature for $\mathrm{S} / \mathrm{F}$ bilayer and multilayer structures was calculated in many works $[22,28,29,30,31,32]$. In most theoretical papers it is assumed that the transition to the superconducting state is of a second order, i.e. the order parameter $\Delta$ varies continuously from zero to a finite value with decreasing temperature $T$. However, generally this is not the case.

If the phase transition is of the second order one can linearize the corresponding equations (the Eilenberger or Usadel equations) for the matrix Green's function $\hat{f}$ assuming that $T$ is close to the critical temperature $T_{c}$. This is this case that was considered in most papers on this topic. The critical temperature of an $\mathrm{S} / \mathrm{F}$ structure can be calculated using an equation obtained from the self-consistency condition. Close to the transition temperature $T_{c}$, the self-consistency condition can be linearized in $\Delta$, and in the Matsubara representation it acquires the form (see, e.g. Ref. [24] and references therein)

$$
\ln \frac{T_{c}}{T_{c}^{*}}=\left(\pi T_{c}^{*}\right) \sum_{\omega}\left(\frac{1}{\left|\omega_{n}\right|}-i f_{\omega}\right),
$$

where $T_{c}$ is the critical temperature in the absence of the proximity effect and $T_{c}^{*}$ is the critical temperature when the proximity effect is taken into account. The condensate function in Eq. (4) is the (11) element of the matrix Green's function $\hat{f}_{\omega}$. In the diffusive case the matrix condensate function obeys the linearized Usadel equation

$$
D_{F} \partial^{2} \hat{f}_{F} / \partial x^{2}-2\left(|\omega| \hat{\sigma}_{0}+i h_{\omega} \hat{\sigma}_{3}\right) \hat{f}_{F}=0
$$

in the F-layer and the equation

$$
D_{S} \partial^{2} \hat{f}_{S} / \partial x^{2}-2\left(|\omega| \hat{\sigma}_{0} \hat{f}_{S}+i \hat{\sigma}_{3} \Delta\right)=0
$$

in the S-layer; where $D_{F, S}$ is the diffusion coefficient in the F- or S-layer, $h_{\omega}=h \operatorname{sgn} \omega, h$ is the value of the exchange field. These equations should be supplemented by boundary conditions that near $T_{c}$ have the form (see, for example, $[24,25]$ and references therein)

$$
\gamma_{F, S} \partial \hat{f}_{F, S} / \partial x=-\left(\hat{f}_{S}-\hat{f}_{F}\right),
$$

where $\gamma_{F, S}=2 R_{b} \sigma_{F, S}, R_{b}$ is the $\mathrm{S} / \mathrm{F}$ interface resistance per unit area, $\sigma_{F, S}$ are the conductivities of the F- and S-films in the normal state. The Usadel equation is applicable to systems with a short mean free path $l$, which, in other words, means that the inverse momentum relaxation time $\tau^{-1}$ should be larger than $\max \{h, 2 \pi T\}$ in the ferromagnet and $\tau^{-1}$ should be larger than $T_{c}$ in the superconductor. If these conditions are not met, one has to solve the more complicated Eilenberger equation.

At the first glance, Eqs. (5)-(6) look simple and seem to allow a straightforward solution. However this is not so, because the order parameter $\Delta$ depends 
on $x: \Delta=\Delta(x)$. In order to solve these equations, a single-mode approximation has been introduced in several papers [6, 22, 29, 30]. Using this approximation one comes to an interesting non-monotonic dependence of $T_{c}$ on $d_{F}$. A more refined multi-mode method leads to a change of this dependence that can be significant for some values of parameters [33].

If the interface transparency is low, Eqs. (5-6) can be solved. The low transparency limit means that the condition $\left|\kappa_{h} \gamma_{F}\right|<<1$ is fulfilled, where $\kappa_{h}^{2}=2\left(|\omega|+i h_{\omega}\right) / D_{F}$. In this case the condensate function in $\mathrm{S}$ is not affected in the main approximation by the proximity effect and is equal to $\hat{f}_{S}=-i \hat{\sigma}_{3} \Delta /|\omega|$, where $\Delta$ is approximately constant in space. A solution for Eq. (5) can be found easily

$$
f_{ \pm}(x)= \pm \frac{f_{S}}{\gamma_{F, S} \kappa_{ \pm}} \exp \left(-\kappa_{ \pm} x\right)
$$

where $\kappa_{ \pm}=\sqrt{2\left(|\omega| \pm i h_{\omega}\right) / D_{F}}, f_{S}=-i \Delta /|\omega|$ and $f_{ \pm}(x)$ are the (11) and (22) elements of the matrix $\hat{f}_{F}$.

Usually the exchange energy $h$ is much larger than the temperature $T$ and the expression for $\kappa_{ \pm}$shows that the condensate function $f_{ \pm}(x)$ decays in a strong ferromagnet $(h>>T)$ on a rather short length $\xi_{F}=\sqrt{D_{F} / 2 h}$ and experiences oscillations with the same period (to be more precise, the period of oscillations is $2 \pi \xi_{F}$ ). This oscillatory behavior of the condensate function $f(x)$ in $\mathrm{F}$ leads to a non-monotonic dependence of the transition temperature $T_{c}$ on the thickness $d_{F}$ and to oscillations of the critical current in $\mathrm{S} / \mathrm{F} / \mathrm{S}$ Josephson junctions (see below).

We would like to emphasize here an important point. The characteristic length of the oscillations and decay of the condensate function is equal to $\xi_{F}=\sqrt{D_{F} / 2 h}$ only in the diffusive limit $(h \tau<<1)$. In the opposite limit $(h \tau>>1)$ the situation is different: the period of the condensate oscillations in the ferromagnet is $2 \pi v_{F} / h$, whereas the decay length is of the order of the mean free path $l[34,35]$.

One can easily see that the singlet component $f_{3}=\left(f_{+}-f_{-}\right) / 2$ is an even function of $\omega$ and the triplet component $f_{0}=\left(f_{+}+f_{-}\right) / 2$ with the zero total spin $(S=0)$ is an odd function of $\omega$. However, both these components, singlet and triplet with $S=0$, coexists in the ferromagnet over a short length of the order of $\xi_{F}$. In the next Section we will see that in case of a non-monotonic magnetization a triplet component with $S= \pm 1$ arises and penetrates the ferromagnet over much larger distance of order $\xi_{N}$.

Of course, in the more complicated $\mathrm{F} / \mathrm{S} / \mathrm{F}$ structure the critical temperature is also suppressed. However, this suppression depends also on the mutual orientation of the magnetization in the left and right side ferromagnets. This property has lead to the idea to switch the system between the superconducting and normal states by varying the magnetization orientation, Refs. $[6,7]$.

Qualitatively, this effect can be understood as follows. Consider an F/S/F structure with thin F- and S-layers. Assuming that the S/F interfaces are 
highly transparent, one can average the Usadel equation over the thickness. In this case the condensate function is continuous across the $\mathrm{S} / \mathrm{F}$ interfaces, and after averaging one can obtain an equation with an effective order parameter $\Delta_{e f f}=\Delta d_{s} / d$ and an effective exchange field [36] $h_{e f f}=\left(h_{l}+h_{r}\right) d_{F} / d$, where $d=2 d_{F}+d_{S}$ is the total thickness of the trilayer and $h_{l}$ and $h_{r}$ are the exchange fields from the left and from the right, respectively. Thus, the F/S/F structure is similar to a magnetic superconductor with an effective exchange field $h_{\text {eff }}$. It is known that the critical temperature of this superconductor decreases with increasing $h_{\text {eff }}$ and may be even a multivalued function of $h_{e f f}$ [37]. If the magnetizations of the left and right side ferromagnet are opposite to each other, we obtain $h_{\text {eff }}=0$ and therefore $T_{c}$ is larger than in the case of parallel orientations of the magnetization when $h_{e f f} \neq 0$. In order to find $T_{c}$ in the general case, one has to solve Eqs. (5-6) with the boundary conditions (7). These calculations have been performed in Refs. [6, 7].

The oscillations of the condensate function in the ferromagnet, Eq. (8), lead to interesting peculiarities not only in the dependence $T_{c}\left(d_{F}\right)$ but also in the Josephson effect in the $\mathrm{S} / \mathrm{F} / \mathrm{S}$ junctions. It turns out that under certain conditions the Josephson critical current $I_{c}$ changes sign and becomes negative. In this case the energy of the Josephson coupling $E_{J}=\left(\hbar I_{c} / e\right)[1-\cos \varphi]$ has a minimum in the ground state when the phase difference $\varphi$ is equal not to 0 , as in conventional Josephson junctions, but to $\pi$ (the so called $\pi$-junction).

This effect was predicted for the first time by Bulaevskii et al [3]. The authors considered a Josephson junction consisting of two superconductors separated by a region containing magnetic impurities. Later on, the Josephson current was calculated for a S/F/S junction [38]. Recently, this interesting theoretical prediction has been confirmed experimentally $[4,5,39,40]$.

\subsection{Exotic superconductivity in $\mathrm{S} / \mathrm{F}$ structures}

As mentioned in the Introduction, there is strong experimental evidence for the realization of non-BCS type of superconducting states in several highly correlated electron systems like the high- $T_{c}$ cuprates, heavy fermions and Srruthenates. In this section, we will demonstrate that under certain conditions triplet pairing is expected also in S/F systems for an arbitrary superconductor S. In other words, the triplet component can be artificially generated by the exchange field. Before presenting explicit calculations, let us summarize certain general feature of superconducting condensate using symmetry arguments.

Due to anticommutation of the fermionic creation $\psi^{+}$and annihilation $\psi$ operators the condensate function $<\psi_{\alpha}(r, t) \psi_{\beta}\left(r^{\prime}, t^{\prime}\right)>$ must be at equal times, $t=t^{\prime}$, an odd function with respect to permutations $\alpha \leftrightarrows \beta, r \leftrightarrows r^{\prime}$. The triplet pairing means that the spins of the Cooper pairs are parallel to each other and the transposition of the spin indices does not change the condensate function. Provided this function remains finite at $t=t^{\prime}$ it must change the sign under transposition of the coordinates $r$ and $r^{\prime}$. So, the triplet 
Cooper pair has to be an odd function of the orbital momentum or, in other words, the orbital angular momentum $L$ is an odd number: $L=1$ (p-wave), 3 etc.

The dependence of the condensate function on the direction in the space makes such a superconductivity very sensitive to disorder. The p-wave condensate (as well as d-wave pairing, etc.) is strongly suppressed by already non-magnetic impurities. Of course, order parameter $\Delta_{\alpha \beta}=\sum_{k} \Delta_{\alpha \beta}\left(\mathbf{k}_{\mathbf{F}}\right) \sim$ $\sum_{k}<\psi_{\alpha}(r, t) \psi_{\beta}\left(r^{\prime}, t\right)>_{k}$ is also suppressed. The s-wave $(L=0)$ singlet condensate is an exception because it is a scalar therefore is not destroyed by non-magnetic impurities (Anderson theorem).

At first glance, any non-singlet pairing should be suppressed by the impurities, which makes an experimental observation very difficult. However, one more a very non-trivial possibility for triplet pairing exists. The previous conclusion about the antisymmetricity of the orbital part of the condensate function remains finite at equal times, which excluded functions antisymmetric in $t-t^{\prime}$.

At the same time, nothing forbids the function $<\psi_{\alpha}(r, t) \psi_{\beta}\left(r^{\prime}, t\right)>$ to change sign under the transposition $t \leftrightarrows t^{\prime}$. In the frequency representation, this property is realized if the correlator $<\psi_{\alpha}(r, \tau) \psi_{\beta}\left(r^{\prime}, \tau^{\prime}\right)>_{k, \omega}$ is an odd function of the Matsubara frequency $\omega$. However, if the condensate function is odd in frequency, it may be even in the momentum and we come to the triplet pairing again. In this case, the correlation function $<\psi_{\alpha}(r, \tau) \psi_{\beta}\left(r^{\prime}, \tau^{\prime}\right)>_{k, \omega}$ equals zero at coinciding times (the sum over all frequencies is zero) and therefore the Pauli principle for the equal-time correlators is not violated.

This type of pairing was suggested by Berezinskii [41] as a possible mechanism of superfluidity of ${ }^{3} \mathrm{He}$. He assumed that the order parameter $\Delta(\omega) \propto$ $\sum_{\omega, k}<\psi_{\alpha}(r, \tau) \psi_{\beta}\left(r^{\prime}, \tau^{\prime}\right)>_{k, \omega}$ is an odd function of $\omega: \Delta(\omega)=-\Delta(-\omega)$. However experiments on superfluid ${ }^{3} \mathrm{He}$ have shown that the Berezinskii's state was not realized in this system. Now it is well known that the condensate in ${ }^{3} \mathrm{He}$ is antisymmetric in the momentum space and symmetric (triplet) in the spin space. Thus, the Berezinskii hypothetical pairing mechanism remained unrealized for few decades.

Recent theoretical studies have shown that a superconducting state similar to the one suggested by Berezinskii might be induced in S/F systems due to the proximity effect $[42,43]$. In the next sections we will analyze this new type of superconductivity with triplet pairing that is odd in frequency and, in the diffusive limit, even in momentum. This can be s-wave pairing and therefore this type of the superconductivity is not sensitive to impurities.

We note, however, that there is a qualitative difference between this new superconducting state in $\mathrm{S} / \mathrm{F}$ structures and the one proposed by Berezinskii. In $\mathrm{S} / \mathrm{F}$ structures both singlet and triplet types of the condensate coexist and the order parameter $\Delta$ existing only in the $\mathrm{S}$ region (we assume that the superconducting coupling in the F region is zero) is determined solely by the singlet part of the condensate. 
Note that, while theories of unconventional superconductivity often imply strongly correlated systems, the triplet state induced in S/F structures can be derived within the framework of the BCS theory valid in the weak-coupling limit. This fact not only drastically simplifies theoretical considerations but also helps in designing experiments, since well known elemental superconductors prepared under controlled growth procedures may be used in order to detect the triplet superconductivity.

To finish this subsection let us summarize the properties of this new type of superconductivity that we call odd triplet superconductivity:

- It contains a triplet component. In particular the components with projection $S= \pm 1$ are insensitive to the presence of an exchange field and therefore long-range proximity effects arise in $\mathrm{S} / \mathrm{F}$ structures.

- In the dirty limit it has an s-wave symmetry. The condensate function is even in $\mathbf{p}$ and therefore, contrary to the unconventional superconductors with triplet pairing, is not destroyed by the presence of non-magnetic impurities.

- The triplet condensate function is odd in frequency.

Before we turn to a more detailed theoretical analysis of the triplet superconductivity, we remark that in the F-regions of the S/F structures no attractive electron-electron interaction exists, and therefore $\Delta=0$ in F. This means that only the superconducting condensate function $f$ in the ferromagnet exists and, as it will become clear later, it arises only due to the proximity effect.

\subsection{Triplet Odd Superconductivity induced by an inhomogeneous magnetization in $\mathrm{S} / \mathrm{F}$ structures}

As discussed in 2.1, the presence of an exchange field results in formation of the triplet component of the condensate function. In a homogeneous exchange field, only the component with the projection $S=0$ is induced. Then the natural question arises: Can the other components with $S= \pm 1$ also be induced? If they could, this would lead to a long range penetration of the superconducting correlations into the ferromagnet because these components correspond to the correlations of the type $\left\langle\psi_{\uparrow} \psi_{\uparrow}\right\rangle$ with parallel spins and they are not as sensitive to the exchange field as the other ones.

In what follows, we analyze a few examples of the $\mathrm{S} / \mathrm{F}$ structures in which all the projections of the triplet component are induced. The common feature of these structures is that the magnetization should be non-homogeneous.

\section{F/S/F trilayer structure}

We start with considering the F/S/F system shown schematically in Fig. 1. The structure consists of one S-layer and two F-layers with the magnetization 


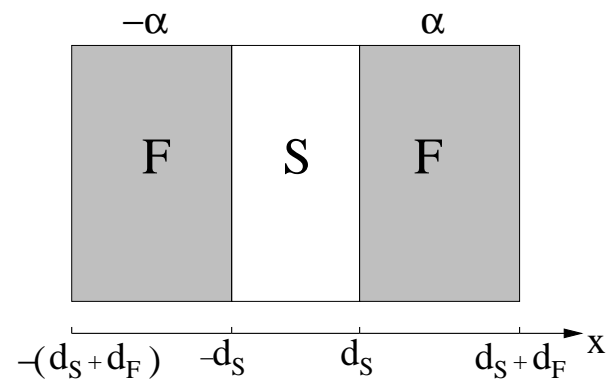

Fig. 1. Trilayer geometry. The magnetization of the left (right) side F-layer makes an angle $\alpha(-\alpha)$ with the $z$-axis.

inclined at the angle $\pm \alpha$ with respect to the $z$-axis (in the $y z$ plane). As we have seen in the previous section, each of the layers generates the triplet component with the zero total projection of the spin, $S=0$, in the direction of the exchange field. If the magnetic moments of the layers are collinear (parallel or antiparallel), the total projection remains zero. However, if the moments of the ferromagnetic layers are not collinear, the superposition of the triplet components coming from the different layers should have all the possible projections of the total spin.

From this qualitative argument we can really expect the non-trivial effect of the generation of the triplet components with all the projections of the total spin provided the thickness of the S-layer is not too large. The point is that the triplet component decays in $\mathrm{S}$ on a length of the order of the coherence length $\xi_{S} \approx \sqrt{D_{S} / \pi T_{c}}$, Eq. (6). We assume that the thickness of the S-layer does not exceed much this length.

In order to find all types of the condensate (singlet and triplet), one has to solve the linearized Usadel equation in the F-region (we assume a weak proximity effect) [43] for the condensate function $\check{f}$ that is a $4 \times 4$ matrix in the particle-hole and spin spaces

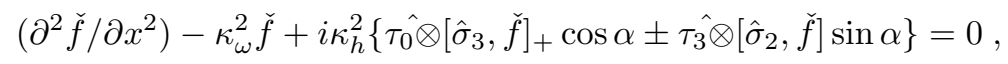

where $\left[\hat{\sigma}_{3}, \check{f}\right]_{+}=\hat{\sigma}_{3} \otimes \check{f}+\check{f} \otimes \hat{\sigma}_{3}$.

The wave vectors $\kappa_{\omega}$ and $\kappa_{h}$ entering Eq. (9) have the form

$$
\kappa_{\omega}^{2}=2|\omega| / D_{F}, \quad \kappa_{h}^{2}=2 h \operatorname{sgn}(\omega) / D_{F}
$$

The magnetization vector $\mathbf{M}$ lies in the $(y, z)$-plane and has the components: $\mathbf{M}=M\{0, \pm \sin \alpha, \cos \alpha\}$. The sign "+" ("-") corresponds to the right (left) side F-film. We consider here the simplest case of a highly transparent $\mathrm{S} / \mathrm{F}$ interface and temperatures close to the superconducting transition temperature $T_{c}$. In this case the function $\check{f}$, being small, obeys a linear equation in $\mathrm{S}$ similar to Eq. (6).

The boundary conditions at the S/F interfaces are obtained by a generalization of Eqs. (7) (see Ref. [43]). 
A solution for Eqs. (9) can easily be found. The matrix $\check{f}$ can be represented as

$$
\check{f}=i \hat{\tau}_{2} \otimes \hat{f}_{2}+i \hat{\tau}_{1} \otimes \hat{f}_{1},
$$

where $\hat{f}_{1}=b_{1}(x) \hat{\sigma}_{1}, \quad \hat{f}_{2}=b_{3}(x) \hat{\sigma}_{3}+b_{0}(x) \hat{\sigma}_{0}$.

For the left side F-layer the functions $b_{k}(x)$ are to be replaced by $\bar{b}_{k}(x)$. For simplicity we assume that the thickness of the F-films $d_{F}$ exceeds $\xi_{F}$ (the case of an arbitrary $d_{F}$ was analyzed in [43]). Using the representation, Eq. (11), we find the functions $b_{i}(x)$ and $\bar{b}_{i}(x)$. They are decaying exponential functions and can be written as

$$
b_{k}(x)=b_{k} \exp \left(-\kappa\left(x-d_{S}\right)\right), \quad \bar{b}_{k}(x)=\bar{b}_{k} \exp \left(\kappa\left(x+d_{S}\right)\right) .
$$

Substituting Eq. (12) into Eq. (9), we obtain a set of linear equations for the coefficients $b_{k}$ that should be complemented by expressions for the eigenvalues $\kappa$.

In the limit of large exchange energy $h\left(\{T, \Delta\}<<h\right.$, but $\left.h<<\tau^{-1}\right)$, the eigenvalues $\kappa$ are equal to

$$
\kappa=\kappa_{\omega}, \quad \kappa_{ \pm} \approx(1 \pm i) \kappa_{h}
$$

We see from Eq. (13) that the solutions $\kappa_{\omega}$ and $\kappa_{ \pm}$are completely different. The roots $\kappa_{ \pm}$proportional to $\kappa_{h}$ are very large and therefore the corresponding solutions $b_{k}(x)$ decay very fast (similar to the singlet component). This is the solution that exists for a homogeneous magnetization (collinear magnetization vectors).

In contrast to the roots $\kappa_{ \pm}$, the value for $\kappa_{\omega}$ given by Eq. (13) does not depend on the exchange energy $h$ and is much smaller. It is this eigenvalue that leads a to slow decay of the superconducting correlations. The solutions corresponding to the root $\kappa_{\omega}$ describe the long-range penetration of the triplet component into the ferromagnetic region. The function $b_{1}(x)$ is the amplitude of the triplet component penetrating the F-region over a long distance of the order of $\kappa_{\omega}^{-1} \sim \xi_{N}$. Its value as well as the values of the other functions $b_{k}(x)$ is to be found from the boundary conditions at the $\mathrm{S} / \mathrm{F}$ interfaces.

Matching the solutions in $\mathrm{S}$ and $\mathrm{F}$ at the $\mathrm{S} / \mathrm{F}$ interfaces, we obtain the coefficients $b_{k}$ and $\bar{b}_{k}$. Note that $b_{3 \pm}=\bar{b}_{3 \pm}$ and $b_{\omega}=-\bar{b}_{\omega}$. Although the solution can be found for arbitrary parameters entering the equations, we present here the expressions for $b_{3 \pm}$ and $b_{\omega}$ in some limiting cases only. For example, if the parameter $\gamma \kappa_{h} / \kappa_{S}$ is small, the amplitudes of the long-range triplet component $b_{\omega}$ and singlet components $b_{3 \pm}$ can be written in a rather simple form

$$
b_{\omega} \approx-\frac{2 \Delta}{E_{\omega}}\left(\frac{\gamma \kappa_{h}}{\kappa_{S}}\right) \frac{\sin \alpha \cos ^{2} \alpha}{\sinh \left(2 \Theta_{S}\right)}, \quad b_{3+} \approx b_{3-} \approx \frac{\Delta}{2 i E_{\omega}}
$$

where $\Theta=\kappa_{S} d_{S}$ and $E_{\omega}=\sqrt{\omega^{2}+\Delta^{2}}, \gamma=\sigma_{F} / \sigma_{S}$ and $\sigma_{F}\left(\sigma_{S}\right)$ is the conductivity in the ferromagnet (superconductor). 
At the $\mathrm{S} / \mathrm{F}$ interface the amplitude of the triplet component $b_{\omega}$ is small compared to the magnitude of the singlet one $b_{3+}$. However, the triplet component decays over a long distance $\xi_{N}$, while the singlet one vanishes at distances exceeding the short length $\xi_{F}$. The amplitudes $b_{\omega}$ and $b_{3 \pm}$ become comparable if the parameter $\gamma \kappa_{h} / \kappa_{S}$ is of the order of unity.

It follows also from Eq. (14) that the amplitude of the triplet component $b_{\omega}$ is zero in the case of collinear vectors of magnetization, i.e. at $\alpha=0$ or $\alpha=\pi / 2$. It reaches the maximum at the angle $\alpha_{m}$ for which $\sin \alpha_{m}=1 / \sqrt{3}$. Therefore the maximum angle-dependent factor in Eq. (14) is $\sin \alpha_{m} \cos ^{2} \alpha_{m}=2 / 3 \sqrt{3} \approx 0.385$.

One can see from Eq. (14) that $b_{\omega}$ becomes exponentially small if the thickness $d_{S}$ of the S-films significantly exceeds the coherence length $\xi_{S} \approx$ $\sqrt{D_{S} / \pi T_{c}}$. This means that in order to have a considerable penetration of the superconducting condensate into the ferromagnet, one should not make the superconducting layer too thick. On the other hand, if the thickness $d_{S}$ is too small, the critical temperature $T_{c}$ is suppressed. In order to avoid this suppression, one has to use, for instance, a F/S/F structure with a small thickness of the F-films. In Fig. 2 we plot the spatial dependence of the singlet and triplet components in $\mathrm{F} / \mathrm{S} / \mathrm{F}$ structure.

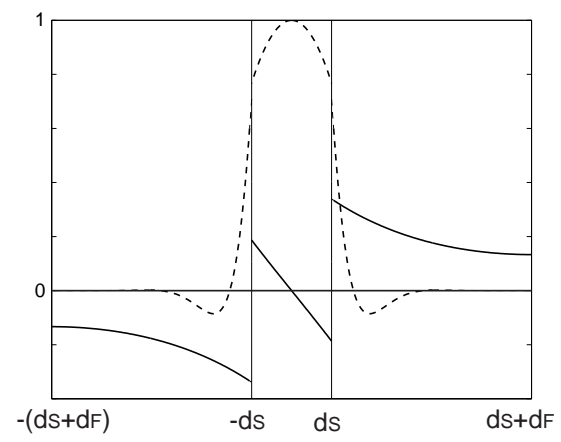

Fig. 2. The spatial dependence of $\operatorname{Im}\left(b_{3}(x)\right)$ (dashed line) and the long-range part of $\operatorname{Re}(b(x))$ (solid line). We have chosen $\sigma_{F} / \sigma_{S}=0.2, h / T_{c}=50, \sigma_{F} R_{b} / \xi_{F}=$ $0.05, d_{F} \sqrt{T_{c} / D_{S}}=2, d_{S} \sqrt{T_{c} / D_{S}}=0.4$ and $\alpha=\pi / 4$. The discontinuity of the triplet component at the $\mathrm{S} / \mathrm{F}$ interface is because the short-range part is not shown in this figure. Taken from [43].

\section{Domain wall at the S/F interface and helical ferromagnets}

Now we consider another example of an S/F structure in which the long-range triplet component (LRTC) also arises. This structure is shown schematically in Fig. 3. It consists of an $\mathrm{S} / \mathrm{F}$ bilayer with a non-homogeneous magnetization in the F-layer. We assume for simplicity that the magnetization vector $\mathbf{M}=$ 


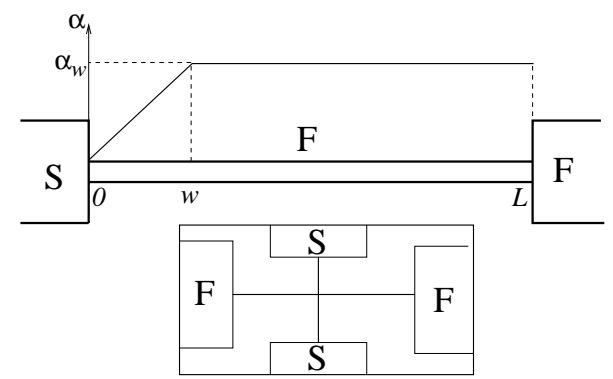

Fig. 3. S/F structure with a domain wall in the region $0<x<w$. In this region $\alpha=Q x$, where $Q$ is the wave vector which describes the spiral structure of the domain wall. For $x>w$ it is assumed that the magnetization is homogeneous, i.e., $\alpha=Q w$.

$M(0, \sin \alpha, \cos \alpha)$ rotates in the F-film starting from the $\mathrm{S} / \mathrm{F}$ interface $(x=0)$, and the rotation angle has a simple, piece-wise $x$-dependence: $\alpha(x)=Q x$, in the domain wall for $0<x<w$ and $\alpha(x)=Q w$ for $w<x$. This means that the $\mathbf{M}$ vector is directed parallel to the $z$-axis at the $\mathrm{S} / \mathrm{F}$ interface and rotates by the angle $\alpha(w)$ over the length $w$ ( $w$ may be the width of a domain wall). At $x>w$ the orientation of the vector $\mathbf{M}$ is fixed. This structure was considered first in [42] and later in [44]. The Usadel equation for this case has been solved in Ref. [42]. The solution is found in the region of the domain wall $0<x<w$ and in the region of a constant magnetization: $w<x<\infty$.

For this configuration of the magnetic moment the LRTC arises in the domain wall and spreads into the ferromagnet over a long distance. The characteristic decay length of the LRTC inside the domain wall is

$$
\xi_{Q}=\left(Q^{2}+\kappa_{\omega}^{2}\right)^{-1 / 2}
$$

whereas its value equals $\kappa_{\omega}^{-1}$ outside the domain wall. The singlet component penetrates the ferromagnet over a short length of the order of $\xi_{F}$. Although the amplitude of the LRTC at the S/F interface may be comparable with the amplitude of the singlet component, the decay length of the LRTC is much larger (see Fig. 4). One more system where the LRTC arises is a helical ferromagnet [45] (see Fig. 15). Such a structure is realized, for example, in several heavy rare earth metals. In this ferromagnet the magnetization vector rotates around the $z$-axis and has a non-zero projection $H_{z}$ on this axis.

It was shown that in this case the LRTC penetrates the ferromagnet over a length of the order of $\xi_{Q}$, Eq. (15). What is interesting, the monotonic decay of the LRTC in this case occurs only if the the cone angle $\theta$ is less than $\sin ^{-1}(1 / 3) \approx 19^{\circ}$. At larger $\theta$ the decay of the LRTC is accompanied by oscillations. In the quasi-ballistic case $(h \tau>1)$, the characteristic length of the LRTC penetration into the ferromagnet changes.

In the case of Neel-type domain walls the LRTC vanishes provided the magnetization vector $M$ rotates continuously [46]. However, in an S/F struc- 


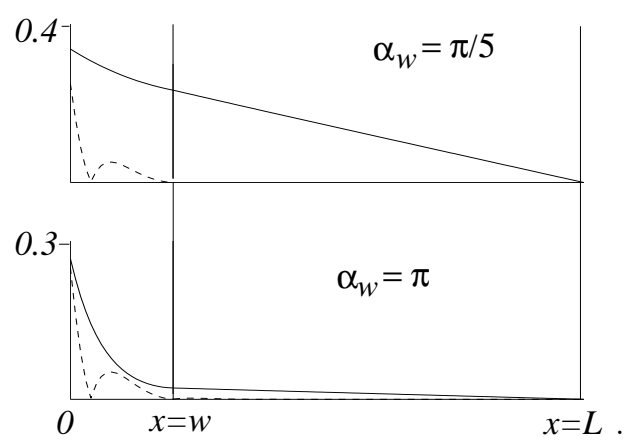

Fig. 4. Spatial dependence of amplitudes of the singlet (dashed line) and triplet (solid line) components of the condensate function in the $\mathrm{F}$ wire for different values of $\alpha_{w}$. Here $w=L / 5, \epsilon=E_{T}$, and $h / E_{T}=400 . E_{T}=D_{F} / L^{2}$ is the Thouless energy, $\epsilon=i \omega$ is the energy (From [42])

ture with several Neel domain walls (the vector $M$ rotates only inside the domain walls) the LRTC arises at the domain walls and decays in the domains over a large distance [47].

\subsection{Other proximity effects in $\mathrm{S} / \mathrm{F}$ structures}

Up to now we implicitly assumed that the proximity effect in S/F structures changes the superconducting properties but leaves the magnetization of the Flayer unchanged. However, this is not always true and experiments performed by [48] and [49] indicate that the ferromagnetic magnetization of S/F bilayers may decrease when lowering the temperature below the superconducting transition temperature $T_{c}$. At that time it was not quite clear what physical mechanism causes this decrease of the magnetization. Here we review two different and independent mechanisms that may explain the effect.

\subsection{Cryproferromagnetism}

In a classic paper Anderson and Suhl [16] proposed an idea that at some circumstances superconductivity might coexist with a non-homogeneous magnetic ordering. They called this magnetic non-homogeneously ordered state cryptoferromagnetic. The basic reasoning leading to this suggestion was that superconductivity could survive in a ferromagnetic background, if the magnetization direction varied on a scale smaller than the superconducting coherence length. The cryptoferromagnetic state in $\mathrm{S} / \mathrm{F}$ structures was considered first in Ref. [17] in the case of a weak ferromagnet.

In a more recent theoretical paper on cryptoferromagnetism in $\mathrm{S} / \mathrm{F}$ bilayers [18] a more realistic case of a strong ferromagnet was considered. It was shown that even if the exchange field is large the cryptoferromagnetic state is still possible provided the ferromagnetic film is sufficiently thin. A 
phase diagram containing the cryptoferromagnetic state has been drawn depending on the stiffness of the ferromagnet $\mathcal{J}$, the thickness of the F-film $d_{F}$ and the exchange field $h$ of the system. This phase diagram $(a, \lambda)$ for the $\mathrm{S} / \mathrm{F}$ system is represented in Fig. 5 , where $a=2 h^{2} d_{F}^{2} /\left(D_{F} T_{c} \eta^{2}\right), \eta=v_{F} / v_{S}$, $\lambda=\left(\mathcal{J} d_{F} / N_{F} \sqrt{2 T_{c} D_{F}^{3}}\right)\left(7 \varsigma(3) / 2 \pi^{2}\right)$, and $N_{F}$ is the density-of-states (DOS) in the ferromagnet. Estimates of the parameters $\left(\mathcal{J}, h\right.$ and $\left.d_{F}\right)$ for the samples

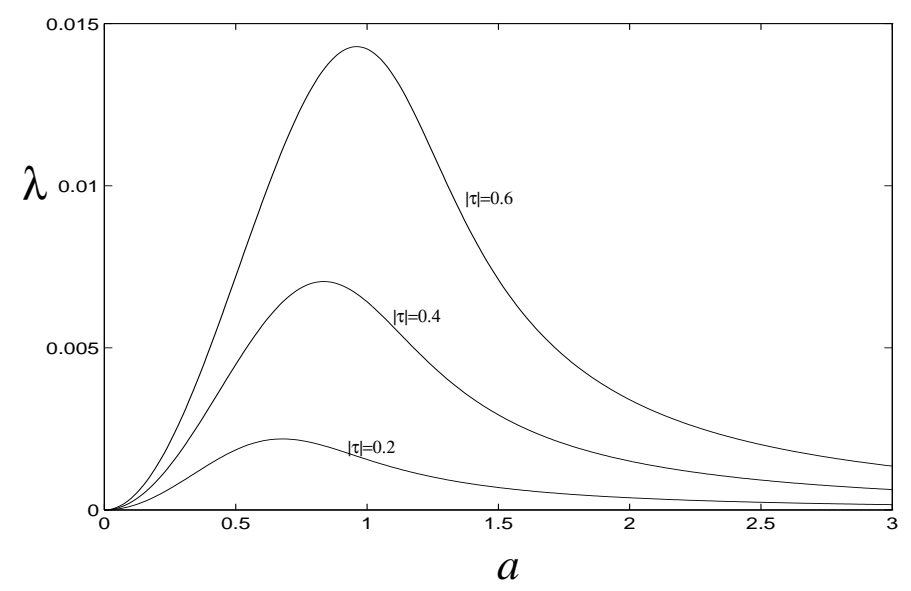

Fig. 5. Phase diagrams $(\lambda, \alpha)$ for different values of $|\tau|=\left(T_{c}-T\right) / T_{c}$. The area above (below) the curves corresponds to the $\mathrm{F}(\mathrm{CF})$ state.

used in the experiments [49] in which a reduction of the effective magnetization was observed show that the results of Ref. [18] agree with the experimental data.

The calculations show that the proximity effect may lead to a magnetic spiral structure in the F-film even if the exchange energy $h$ is much larger than the characteristic energy of the superconducting transition $T_{c}$. This cryptoferromagnetic ordering is related to existence of low lying states in the ferromagnet. The spiral structure increases the magnetic energy only by a small amount, whereas the energy of interaction between the exchange field and the superconductivity can essentially be reduced.

At the same time, there exists another mechanism that can reduce the total magnetization in $\mathrm{S} / \mathrm{F}$ structure and it is also due to the proximity effect. This is the so-called inverse proximity effect describing the situation when the orientation of the magnetization remains unchanged, while its magnitude changes both in the S- and F-layers.

\subsection{Inverse proximity effect}

The inverse proximity effect is due to a contribution of free electrons both in the ferromagnet $\left(\delta M_{F}\right)$ and in the superconductor $\left(M_{S}\right)$ to the total magne- 
tization. On one hand, the DOS in the F-film is reduced due to the proximity effect, thus decreasing the magnetization in $\mathrm{F}$ by $\delta M_{F}$. On the other hand, the Cooper pairs in $\mathrm{S}$ are polarized in the direction opposite to $M_{F}$, giving rise to a magnetization $\left(M_{S}\right)$ with a direction opposite to $M_{F}$. So, the S-layer becomes ferromagnetic and this is the reason for calling this effect the inverse proximity effect. For a more detailed qualitative explanation of this mecha-

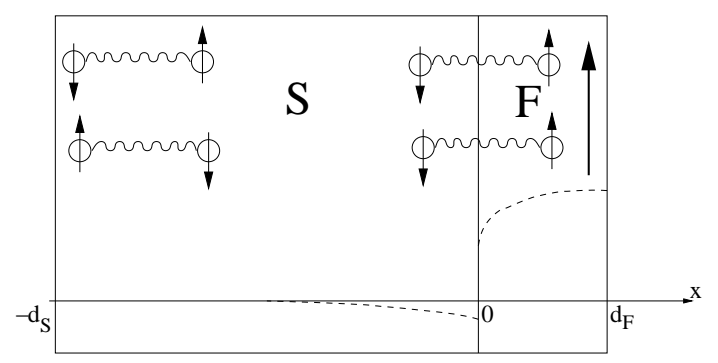

Fig. 6. S/F structure and schematic representation of the inverse proximity effect. The dashed curves show the local magnetization.

nism we consider the $\mathrm{S} / \mathrm{F}$ structure with a thin F-layer in Fig. 6. We assume that the exchange field of $\mathrm{F}$ is homogeneous and directed along the $z$-axis.

If temperature exceeds the critical temperature $T_{c}$, the total magnetization of the system $M_{t o t}$ equals $M_{0} d_{F}$, where $d_{F}$ is the thickness of the F-layer. When the temperature is lowered below $T_{c}$, the S-layer becomes superconducting and Cooper pairs with a size of the order of $\xi_{S}$ appear in the superconductor. Due to the proximity effect the Cooper pairs cross the interface and penetrate into the ferromagnet. In the case of a homogeneous magnetization in $\mathrm{F}$ the Cooper pairs are composed of electrons with opposite spins, such that the total magnetic moment of the pair equals zero. The exchange field is assumed to be not too strong, otherwise the Cooper pairs would be destroyed.

It is clear from this simple picture that pairs located entirely in the superconductor cannot contribute to the magnetic moment of the superconductor. However, some pairs are distributed in space in a more complicated manner: one of the electrons of the Cooper pair stays in the superconductor, whereas the other one enters the ferromagnet. These are these pairs that create the magnetic moment in the superconductor.

Energetically it is favorable for the electron of the Cooper pair with the spin parallel to the magnetization of the ferromagnet to have a higher probability density in F. This means that the electron with the opposite spin has a higher probability density in S. This is the reason why these pairs form a magnetic moment in the S-layer. As a result, the ferromagnetic order is created in the S-layer with a direction of the magnetic moment opposite to the direction of $\mathbf{M}$ in F-layer. The induced magnetic moment penetrates the 
superconductor over the size of the Cooper pairs, which may be much larger than $d_{F}$.

Using similar arguments we can predict a related effect: the magnetic moment in the ferromagnet should be reduced in the presence of superconductivity because some of the electrons located entirely in the ferromagnet condense into Cooper pairs and do not contribute to the magnetization.

From this qualitative, simplified picture one can expect that the total magnetization of an $\mathrm{S} / \mathrm{F}$ system will be reduced for temperatures below $T_{c}$. A quantitative analysis based on the Usadel equation (diffusive case) [19] or on the Eilenberger equation (quasiballistic case) [50] supports the qualitative picture. It turned out that at low temperatures the magnetic moment $M_{F}$ in $\mathrm{F}$ is screened completely by the spin-polarized Cooper pairs in $\mathrm{S}$ if $M_{F}$ is due to free electrons (ideal itinerant ferromagnet) i.e. $M_{S}=-M_{F}$. This conclusion is valid in the limit $h<D_{F} / d_{F}^{2}$.

With increasing the exchange energy $h$ the induced magnetic moment decreases monotonically in the diffusive limit [19] or non-monotonically in the clean limit [50].

It should be stressed that both the mechanism discussed here and that of the last section lead to a decrease of the total magnetization. The spin polarization of Cooper pairs in the superconductor in $\mathrm{F} / \mathrm{S} / \mathrm{F}$ structure with a non-collinear magnetization in $\mathrm{F}$ was studied in Ref. [51].

\section{$3 \mathrm{~S} / \mathrm{F}$ proximity effect: Experiments}

\subsection{Superconducting transition temperature in $\mathrm{F} / \mathrm{S}$ systems}

Following the theoretical predictions (see Sec.2.2) a ferromagnetic film deposited on a superconducting film should drastically suppress the superconducting $T_{c}$. In experimental systems, however, this is often not the case, the $T_{c^{-}}$suppression appears rather moderate. This is due to two different reasons. First, in real thin film systems there is often an intermediate alloy layer caused by interdiffusion that is weakly magnetic or even non-magnetic. This is the case, e.g., in $\mathrm{Fe} / \mathrm{Nb}[52,53]$ and, probably, in $\mathrm{Gd} / \mathrm{Nb}[54]$. This interlayer prevents the direct contact between the F- and S-layer and weakens the suppression of $T_{c}$. Second, the quantum mechanical transparency of a real $\mathrm{S} / \mathrm{F}$ interface is often quite small, i.e. the coefficient $\gamma_{F, S}$ in Eq. (8) is small and the $T_{c}$-suppression is much weaker than that with an ideally transparent interface.

An interesting feature of the $\mathrm{S} / \mathrm{F}$ proximity effect that has recently been under intensive discussion in literature, is oscillation of the superconducting transition temperature as a function of the F-layer thickness $d_{F}$.

There are quite different physical mechanisms that may cause $T_{c}\left(d_{F}\right)$ oscillations or a non monotonic $T_{c}\left(d_{F}\right)$ behavior. An indirect mechanism, not directly related to the proximity effect, has been observed in $\mathrm{Fe} / \mathrm{Nb}$ bilayers 
$[52,53]$ (Fig. 7c). Here an alloying at the interface leads to a non-ferromagnetic $\mathrm{NbFe}$ interlayer of about $0.7 \mathrm{~nm}$ thickness and therefore the minimum in $T_{c}\left(d_{F}\right)$ just correlates with the onset of ferromagnetism. The explanation of
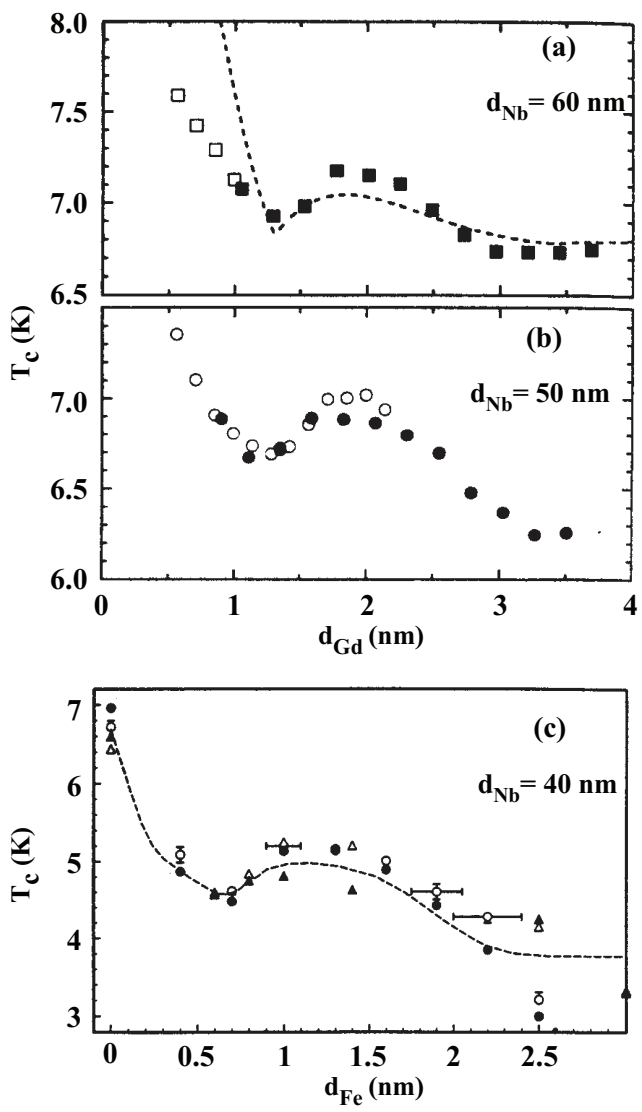

Fig. 7. Dependence of superconducting transition temperature on the ferromagnetic layer thickness in ( $\mathrm{a}$ and $\mathrm{b}$ ) two series of Nb/Gd multilayers ( Jiang et al [54]), and (c) $\mathrm{Fe} / \mathrm{Nb} / \mathrm{Fe}$ trilayers (Mühge et al $[52,53]$ ).

this phenomenon is that strong longitudinal spin fluctuations exist in the $\mathrm{NbFe}$ interlayer with a concentration close to the onset of ferromagnetic long range order. They are responsible for the strong initial $T_{c}$-suppression when increasing the Fe-thickness from 0 to $0.7 \mathrm{~nm}$ in Fig. $7 \mathrm{c}$. When the first ferromagnetic Fe layer appears above $d_{F e}=0.7 \mathrm{~nm}$, the spin fluctuations in the $\mathrm{NbFe}$ interlayer are suppressed by the exchange field of the Fe-layer and result in an increase of $T_{c}$. This is a rather indirect influence of the ferromagnetic Fe-layer on the superconductivity of the Nb layer. 
Now, coming to oscillations in $T_{c}\left(d_{F}\right)$ induced by the $\mathrm{S} / \mathrm{F}$ proximity effect, we recall that, as discussed in Sec. 2.2, oscillations of the condensate function $\hat{f}_{s}$ in space may directly lead to a non-monotonic $T_{c}$-dependence on $h$ or $d_{F}$. Actually the reason for this non monotonic behavior may be different for $\mathrm{S} / \mathrm{F}$ bilayers and S/F/S trilayers, since the boundary conditions in these two cases are different. For bilayers, only one side of the F-layer is in contact with the superconductor, whereas in the S/F/S trilayers the F-layer is in contact with the superconducting layers on the both sides.

For the case of trilayers (but not for bilayers) oscillations of $T_{c}$ may be due to appearance (or disappearance) of Josephson $\pi$-coupling. As mentioned in Sec. 2.3, due to the oscillation of the superconducting pairing function in the F-layer, the phase difference in the superconducting pairing function on both the sides of the F-layer may have opposite phases at certain F-layer thicknesses, i.e., the phase difference between the neighboring S-layers may be equal to $\pi$. Radović et al [28] concluded from their calculations that $T_{c}$ for $\pi$-coupling most probably is higher than for the vanishing phase difference.

Jiang et al [54] claimed that the observed oscillations of $T_{c}\left(d_{G d}\right)$ (Fig. 7a and $7 \mathrm{~b}$ ) are due to this type of the Josephson $\pi$-coupling. Several other works on $\mathrm{S} / \mathrm{F}$ multilayers have reported a single peak in $T_{c}\left(d_{F}\right)$, and have attributed this feature to " $\pi$-switching" (see, e.g., $[55,56,57]$ ).

Whereas the role of $\pi$-coupling for the non monotonous $T_{c}\left(d_{F}\right)$ in $\mathrm{S} / \mathrm{F}$ multilayers has not been finally clarified and an alternative explanation exists (see below), a clear experimental evidence for $\pi$-coupling across an F-layer comes from the study of Josephson junctions using F-layers as barriers [4, 39]. Tunnelling spectroscopy revealed damped oscillations of the superconducting order parameter induced in the F-film by the proximity effect [39]. Ryazanov et al [4] performed measurements of the critical current in Josephson junctions consisting of superconducting $\mathrm{Nb}$ and weakly ferromagnetic interlayers and found that the character of the junction changed from 0-phase at high temperatures to $\pi$-phase at low temperatures. This result has later been confirmed by Blum et al [5]. A different phase sensitive experiment [58] also gave evidence for the oscillatory behavior of the critical supercurrent of S/F layered system when varying the F-layer thickness.

Without invoking the $\pi$-coupling, oscillations of $T_{c}\left(d_{F}\right)$ can simply originate from the oscillations of the condensate amplitude in space within the F-layer. As shown theoretically by [28, 30, 59], due to these oscillations and taking the boundary conditions for the pairing wave function at the S/F interfaces into account, the $T_{c}\left(d_{F}\right)$-curve may have an oscillatory character with the oscillation period of the order $\xi_{h}=v_{F} / h$ (see Sec. 2.2).

The physical origin of the oscillatory character of $T_{c}\left(d_{F}\right)$ can qualitatively be traced back to the propagating character of the superconducting pairing wave function in the ferromagnet. If the thickness of the F-layer is smaller than the penetration depth of the pairing wave function, this function, when transmitted through the S/F interface into the F-layer, will interfere with the wave reflected from the opposite surface of the ferromagnet. As a result, the 
flux of the pairing wave function crossing the $\mathrm{S} / \mathrm{F}$ interface varies with the thickness of the F-layer $d_{F}$.

Then, the coupling between the electrons of the ferromagnet and the superconductor will be modulated and $T_{c}$ will oscillate with $d_{F}$. If the interference at the $\mathrm{S} / \mathrm{F}$ interface is essentially constructive (this corresponds to a minimal jump of the pairing function amplitude at the $\mathrm{S} / \mathrm{F}$ interface), the coupling is weak, and one expects $T_{c}$ to be maximal. When the interference is destructive, the coupling is maximized and $T_{c}\left(d_{F}\right)$ is minimal.

It should be noted that this model explaining $T_{c}\left(d_{F}\right)$ oscillations applies to the case of the $\mathrm{S} / \mathrm{F}$ bilayers as well as to $\mathrm{F} / \mathrm{S} / \mathrm{F}$ trilayers or $\mathrm{S} / \mathrm{F}$ multilayers, whereas the $\pi$-coupling concept does not apply for bilayers.

Aarts et al [60] studied $\mathrm{V} / \mathrm{V}_{1-x} \mathrm{Fe}_{x}$ multilayers without interdiffusion at the interface. They showed that $T_{c}$ strongly depends on the interface transparency and presented experimental evidence for an intrinsically reduced interface transparency. From the dependence of $T_{c}$ on the magnetic layer thickness they calculated the penetration depth of Cooper pairs into the F-layer and found it to be inversely proportional to the effective magnetic moment per Fe atom. For the interpretation of the observed peculiarities they introduced a finite transparency of the S/F interface and argued, based on their experimental data, that with an increasing the exchange splitting of the conduction band in the F-layer the transparency of the S/F interface for Cooper pairs decreases.

Lazar et al [61] studied experimentally the role of the interface transparency in the $\mathrm{Fe} / \mathrm{Pb} / \mathrm{Fe}$ system and, for comparison, in the $\mathrm{Fe} / \mathrm{V} / \mathrm{Fe}$ system, too [62]. In contrast to the case of $\mathrm{Fe} / \mathrm{Nb} / \mathrm{Fe}$ discussed above, in $\mathrm{Fe} / \mathrm{Pb} / \mathrm{Fe}$ and $\mathrm{Fe} / \mathrm{V} / \mathrm{Fe}$ the intermixing at the interfaces is much weaker. Fig. 8 shows the dependence of $T_{c}$ on the thicknesses of the $\mathrm{Fe}$ layers for $\mathrm{Fe} / \mathrm{Pb} / \mathrm{Fe}$ trilayers. A theoretical analysis of the curves using model calculations revealed that the experimental results can only be described assuming $\mathrm{Pb} / \mathrm{Fe}$ interfaces that are not perfectly transparent. The critical temperature $T_{c}$ for the case of $\mathrm{S} / \mathrm{F}$ interfaces with a non-perfect transparency has been calculated by Golubov [60] and Tagirov [30].

A fit to the experimental points using the model calculations [30] is plotted on Fig. 8 as a solid line. The quality of the fit is satisfactory and reproduces the details of the $T_{c}\left(d_{F e}\right)$-curve. The most important parameter obtained from this fit is the value of $T_{m}$, characterizing the transparency of the interface. The fit gives $T_{m}=0.4$. This value corresponds to a quantum mechanical transmission coefficient $\bar{T}=T_{m} /\left(1+T_{m}\right)=0.3[61]$ that is considerably reduced as compared to the ideally transparent interface with $\bar{T}=1$.

Lazar et al [61] concluded that the exchange splitting of the conduction band of the F-layer is the main physical reason for the strongly reduced interface transparency. In principle, the calculation of the interface transparency is a standard quantum mechanical problem of reflection and transmission of electrons at the interface of two metals with different Fermi energies. It is obvious that two electrons with opposite spins forming a Cooper pair can never 


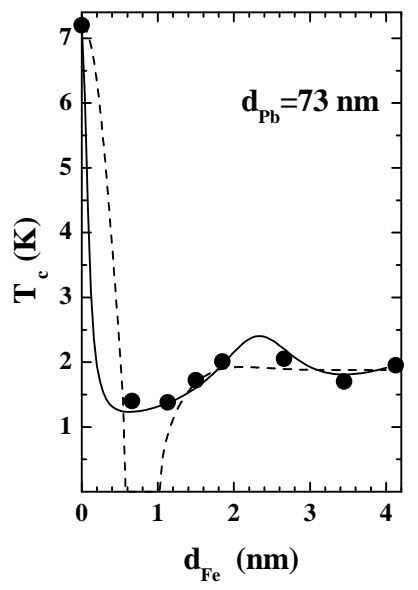

Fig. 8. $T_{c}$ dependence on the Fe thickness at fixed value $d_{P b}=73 \mathrm{~nm}$ for $\mathrm{Fe} / \mathrm{Pb} / \mathrm{Fe}$ trilayers. The dashed line is obtained by the Radović et al [28] theory which supposes an ideally transparent interface. The solid line takes a finite transparency of the interface into account [30].

match the Fermi momenta of the exchange-split subbands of a ferromagnet simultaneously and there will always be a Fermi vector mismatch reducing the transmission. Additionally, a decrease of $\bar{T}$ is expected due to a chemical mismatch of $\mathrm{Pb}$ and $\mathrm{Fe}$ giving rise to a contact potential barrier at the interface. The barrier height should be larger for immiscible metals like $\mathrm{Pb}$ and $\mathrm{Fe}$ in comparison to metals that form solid solutions in the whole concentration range as, e.g., $\mathrm{V}$ and Fe.

Measurements of dependence of $T_{c}$ on the thickness were performed also for $\mathrm{Fe} / \mathrm{V} / \mathrm{Fe}$ trilayers [62]. For two series of samples at small iron thicknesses the transition temperature $T_{c}$ drops sharply when increasing $d_{F e}$ up to $0.5 \mathrm{~nm}$. Then, at $d_{F e} \sim 0.7 \mathrm{~nm}$ for the series with $d_{V}=31$ and $29 \mathrm{~nm}$, a clear minimum of $T_{c}$ is observed. The deepness of this minimum increases with decreasing $d_{V}$. For these two series the residual resistivity ratio $\mathrm{RRR} \simeq 4$, meaning that the mean free path of the conduction electrons in the S-layer $l_{S} \sim 4 \mathrm{~nm}$ [63]. The parameters resulting from a theoretical fit of these curves are the superconducting coherence length $\xi_{S}=\sqrt{\xi_{0} l_{S} / 3.4}=4 \mathrm{~nm}$ (here $\xi_{0}=44 \mathrm{~nm}$ is the BCS coherence length), as estimated from the resistivity data. For the transparency parameter one obtains $T_{m}=1.6$ and for the exchange length in the Fe film $\xi_{h}=0.7 \mathrm{~nm}$.

Using the theoretical model calculations [30] as a guideline, one can extract the important physical parameters necessary for an observation of the theoretically predicted rather spectacular re-entrant behavior of the superconductivity, i.e. superconductivity vanishing for a certain range of $d_{F}$ and coming back for larger $d_{F}$. The system should possess a large electron mean free path in the F- as well as in the S-layer, a high quantum-mechanical transparency 
of the S/F interface and a geometrically flat interface without introducing too much diffuse scattering of the electrons. The last two conditions are well fulfilled in $\mathrm{Fe} / \mathrm{V} / \mathrm{Fe}$ trilayers, so one could try to further increase the electron mean free path $l_{F}$ or $l_{S}$. Whereas this is hardly possible for the F-layers since $l_{F}$ is limited by the very small layer thickness $d_{F}$, improved growth conditions of the V-layer is a promising perspective to increase $l_{S}$ This was accomplished by samples prepared on single crystalline $\mathrm{MgO}$ (100) with nearly epitaxial quality and an RRR-value of the order of 10. For this set of samples clear re-entrant superconductivity was observed (Fig. 9) for the first time. From

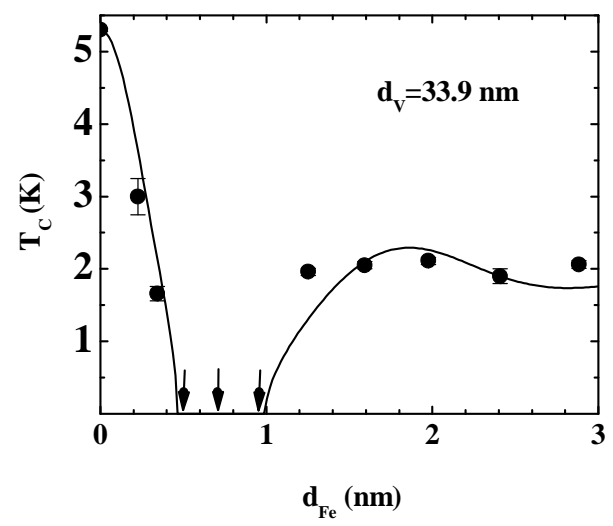

Fig. 9. Superconducting transition temperature vs Fe thickness at fixed $V$ thickness for the $\mathrm{Fe} / \mathrm{V} / \mathrm{Fe}$ series with $d_{V}=33.9 \mathrm{~nm}$. The drawn line is a theoretical curve with the parameters given in the text.

the RRR-value for this series we estimate the mean free path of the conduction electrons $l_{S} \sim 12 \mathrm{~nm}$ and the corresponding coherence length $\xi_{S} \sim 13$ $\mathrm{nm}$. The latter was used for the theoretical fit of the $T_{c}\left(d_{F e}\right)$-curve in Fig. 9. From the transparency parameter $T_{m}=1.6$ the average quantum mechanical transmission coefficient $\bar{T}[61]$ can be estimated to be $\bar{T} \simeq 0.6$. This value of $\bar{T}$ is about twice as large as the $\bar{T}$-value for the $\mathrm{Pb} / \mathrm{Fe}$ interface discussed above [61]. This relatively high transparency of the $\mathrm{Fe} / \mathrm{V}$ interface is an essential ingredient for observing re-entrance behavior. As mentioned above, a highly transparent S/F interface is difficult to achieve with strong ferromagnets, since problems with the matching of the Fermi momentum necessarily occur for at least one spin direction.

An even higher transparency of the $\mathrm{S} / \mathrm{F}$ interface can, in principle, be achieved combining a superconductor with a ferromagnet weakened by dilution. Recently [64], the re-entrant superconductivity has been observed for the $\mathrm{Nb} / \mathrm{Cu}_{1-x} \mathrm{Ni}_{x}$ bilayers.

It is important to note that the results described above for the Fe/V system were fitted using the values of the mean free path of the conduction electrons 
in the F- and S-layers calculated from the resistivity data. In contrast, for the $\mathrm{Nb} / \mathrm{Cu}_{1-x} \mathrm{Ni}_{x}$ system Zdravkov et al [64] had to use surprisingly large values for the mean free path for the conduction electrons in the F-layer in their fitting procedure.

Finishing this section we would like to mention also study of the interplay between magnetism and superconductivity in epitaxial structures of half metal-colossal magnetoresistive $\mathrm{La}_{2 / 3} \mathrm{Ca}_{1 / 3} \mathrm{MnO}_{3}$ (LCMO) and high- $T_{c}$ superconducting $\mathrm{YBa}_{2} \mathrm{Cu}_{3} \mathrm{O}_{7-\delta}$ (YBCO) [65, 66, 67, 68, 69]. Jacob et al [65] demonstrated possibility of preparation of hybrid perovskite high- $T_{c}$ superconductor/ferromagnet superlattices. The superlattices consisting of $\mathrm{YBCO}$ and LBMO $\left(\mathrm{La}_{2 / 3} \mathrm{Ba}_{1 / 3} \mathrm{MnO}_{3}\right)$ layers with the thickness of a few unit cells showed both strong colossal magnetoresistance at room temperature and superconductivity at low temperatures.

Yeh et al [66] reported phenomena manifesting nonequilibrium superconductivity induced by spin-polarized quasiparticles in F/I/S (I is insulator) structures. Sefrioui et al [67], basing on their measurements of $T_{c}$ vs S- and Flayer thickness, speculate that injection of spin-polarized carriers from LCMO into YBCO may add a new source of superconductivity suppression: pair breaking by spin-polarized carriers. This pair breaking effect extends over the spin diffusion length into $\mathrm{S}$ that can be very long (it can be as long as $8 \mathrm{~nm}$ for YBCO). As a result, in the YBCO layer superconductivity is suppressed by the presence of manganite layers with a characteristic length scale much longer than the one predicted by existing theories of the S/F proximity effect.

The same result has been obtained by Holden et al [68] using ellipsometry measurements of the far-infrared dielectric properties of superlattices composed of thin layers of YBCO and LCMO. Finally, Soltan et al [69] studied the role of spin-polarized self injection from LCMO into the YBCO layer. They concluded that the nearly full spin polarization at the Fermi level of LCMO leads to quenching of the proximity effect since it prevents the Cooper pairs from tunnelling into the magnetic layer. Thus, one can see that the results for superlattices consisting of YBCO and LCMO presented above are somewhat contradicting to each other. Nevertheless, they provide an avenue for future theoretical studies of the $\mathrm{F} / \mathrm{S}$ proximity effect in the presence of the spin-polarized ferromagnets.

\subsection{Superconducting spin valve}

In recent years much attention has been devoted to experimental realization of the so-called superconducting spin valve. As described in Sec. 2.1, a consequence of the $\mathrm{S} / \mathrm{F}$ proximity effect is that the superconducting transition temperature of a $\mathrm{F} / \mathrm{S} / \mathrm{F}$ sandwich depends on the mutual orientation of the magnetization of the two F-layers, the antiparallel orientation having a higher $T_{c}$ than the parallel one [6]. In an ideal superconducting spin valve the superconductivity of the S-layer can be switched on and off by rotating the magnetization of one of the F-layers relative to the other, giving an infinite 
magnetoresistance for the switching field. The device is similar to the well known conventional spin valve $\mathrm{F} / \mathrm{N} / \mathrm{F}$ system with a normal metallic layer $\mathrm{N}$ interleaved between two ferromagnetic layers $\mathrm{F}$. In this device the antiparallel magnetization state usually has a larger resistance than the parallel one.

It turned out that the realization of a superconducting spin valve is difficult experimentally and the effects obtained until now are quite small. There are two recent reports in the literature on the successful realization a $\mathrm{F} / \mathrm{S} / \mathrm{F}$ superconducting spin valve. In the $\mathrm{CuNi} / \mathrm{Nb} / \mathrm{CuNi}$ trilayer system $[70,71]$ the maximum shift by only $6 \mathrm{mK}$ of the superconducting transition temperature $T_{c}$ by changing the mutual orientation of the two ferromagnetic layers from parallel to antiparallel was observed. Actually, such a small shift may also be due to changes of the domain structure of the ferromagnetic layers under the influence of the external magnetic field [72].

Pena et al [14] measured the magnetoresistance of $\mathrm{F} / \mathrm{S} / \mathrm{F}$ trilayers combining the ferromagnetic manganite $\mathrm{La}_{0.7} \mathrm{Ca}_{0.3} \mathrm{MnO}_{3}$ with the high- $T_{c}$ superconductor $\mathrm{YBa}_{2} \mathrm{Cu}_{3} \mathrm{O}_{7}$. They observed a magnetoresistance in excess $1000 \%$ for the superconducting state of $\mathrm{YBa}_{2} \mathrm{Cu}_{3} \mathrm{O}_{7}$ that vanished in the normal state.

There is another possible design for the realization of the superconducting spin valve effect proposed by Sungjun Oh et al [73] that found less attention until now. It has the layer structure $\mathrm{S} / \mathrm{F} 1 / \mathrm{N} / \mathrm{F} 2$, i.e. two ferromagnetic layers F1 and F2 separated by a non-magnetic $(\mathrm{N})$ layer are deposited on the one side of the superconductor with $\mathrm{F} 1$ and $\mathrm{N}$ thin enough to allow the superconducting pair wave function to penetrate into F2. The authors have shown that changing the mutual magnetization direction of F1 and F2 from parallel to antiparallel results in a substantial difference $\Delta T_{c}$ when the microscopic parameters for S- and F-films are optimized.

For the realization of the F/S/F spin valve design [6] it would be optimal to use a system where the re-entrant $T_{c}\left(d_{F}\right)$-behavior is observed. As discussed above, $\mathrm{Fe} / \mathrm{V} / \mathrm{Fe}$ fulfills this criterion (see Fig. 9). However, an acceptable performance of the spin valve with a sizable shift of $T_{c}$ can only be expected if the S-layer thickness $d_{S}$ is close to the superconducting coherence length $\xi_{S}$. The studies of the $\mathrm{Fe} / \mathrm{V} / \mathrm{Fe}$ system however revealed that the superconductivity vanishes typically already at $d_{S}<3 \xi_{S}$. A possibility to overcome this problem and maintain superconductivity at $d_{S} \sim \xi_{S}$ is to introduce very thin non-ferromagnetic layers between the S- and F-layers that should screen to some extent the very strong exchange field of the F-layers.

A proper $\mathrm{Fe} / \mathrm{Cr} / \mathrm{V} / \mathrm{Cr} / \mathrm{Fe}$ system, where the $\mathrm{Cr}$ layers play the role of such screening layers, has been studied in detail [74]. In Fig. 10 the $T_{c}$ values measured for the samples from series with a fixed $d_{F e}=5 \mathrm{~nm}$ and $d_{C r}$ varied are plotted. In other three series $d_{C r}$ has been kept constant at $d_{C r}=1.5,2.8$ and $4.7 \mathrm{~nm}$ and the thickness of the Fe-layer was varied. The results for the transition temperatures of these series are reproduced in Figs. 11b-11d and compared to previous results on Fe/V/Fe trilayers [62] (Fig. 11a). The salient features of the results shown in Fig. 11 are as follows. 


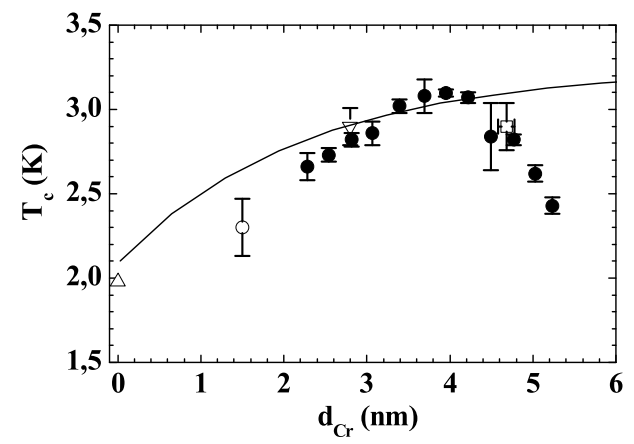

Fig. 10. The superconducting transition temperature as a function of the Cr-layer thickness for all samples from series (1). The solid line is a theoretical curve (see main text).

(1) The overall shape of the $T_{c}\left(d_{F e}\right)$-curve is similar to that obtained for $\mathrm{Fe} / \mathrm{V} / \mathrm{Fe}$.

(2) The amplitude of the initial drop in $T_{c}$ decreases with increasing the thickness of the interleaved Cr-layer.

(3) At $d_{C r}=4.7 \mathrm{~nm}$ in Fig. 11d the Fe-layers have virtually no influence on $T_{c}$ any more, indicating that the amplitude of the pair wave function in the Fe-layer is negligible. This allows the estimation of the penetration depth of the pair wave function in $\mathrm{Cr}$ of about $4 \mathrm{~nm}$, consistent with the results on $\mathrm{Cr} / \mathrm{V} / \mathrm{Cr}$ trilayers [75]. These features are due to the expected screening effect of the Cr-layer, since with increasing $d_{C r}$ the Cooper pair density reaching the Fe-layer is continuously reduced and the effect of the strong exchange field in Fe on the superconductivity is weakened.

The results of the model calculations are shown by the solid lines in Figs. 10 and 11a-11d and neglect the complications caused by the spin density wave (SDW) state of antiferromagnetic Cr. The standard procedure described in the literature (see, e. g., [75] and references therein) was applied and the proximity effect of the $\mathrm{V} / \mathrm{Cr}$ interface was treated by the conventional theory for $\mathrm{S} / \mathrm{N}$ metal films originally developed by de Gennes [1]. In addition, pair breaking scattering of Abrikosov-Gor'kov type [76] at magnetic defects in the Cr-layer is characterized by a spin-flip scattering time $\tau_{s}$ i.e. $\mathrm{Cr}$ is treated as a paramagnetic $(\mathrm{P})$ layer.

Theory of the proximity effect for $\mathrm{S} / \mathrm{P} / \mathrm{F}$ layer system has been developed by Vodopyanov et al [77]. With certain assumptions [74] and the microscopic 


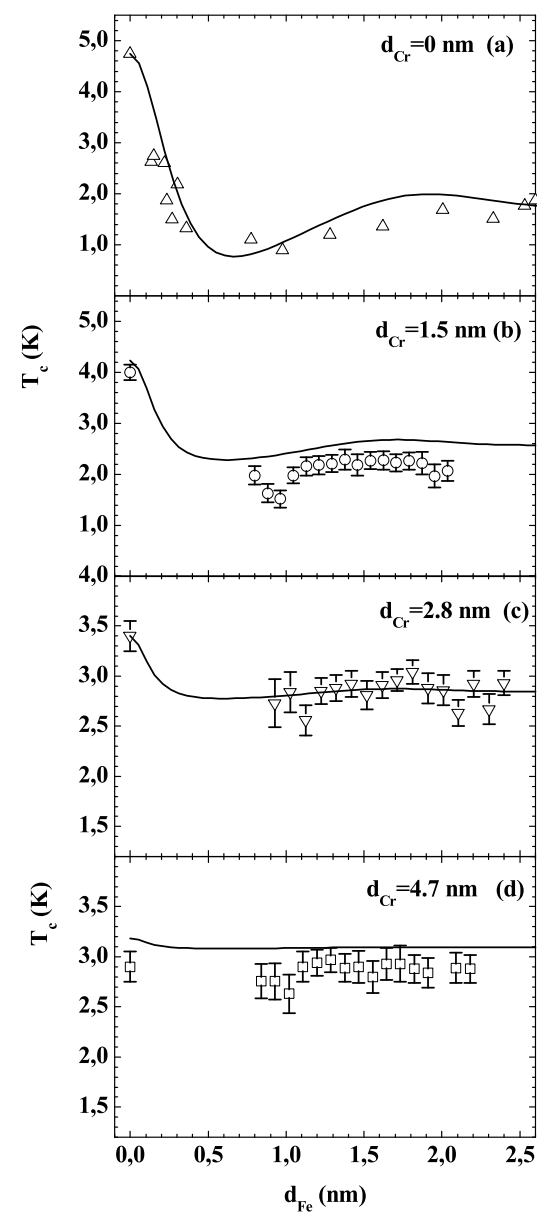

Fig. 11. Superconducting transition temperature as a function of the Fe-layer thickness for samples from series with $d_{C r}=1.5 \mathrm{~nm}(\mathrm{~b})$; with $d_{C r}=2.8 \mathrm{~nm}$ (c); with $d_{C r}$ $=4.7(\mathrm{~d})$. The corresponding curve for $\mathrm{Fe} / \mathrm{V} / \mathrm{Fe}$ trilayers is taken from Ref. [62] and shown in (a) for comparison. The solid lines are calculations according to a model explained in the main text.

parameters known from the studies of the $\mathrm{Fe} / \mathrm{V} / \mathrm{Fe}$ trilayers [62] all data points in Figs. 10 and 11 have been fitted simultaneously, with $\tau_{s}$ being the only fitting parameter. All curves can be best described with $\tau_{s}=5 \cdot 10^{-13} \mathrm{~s}$. 
The overall shape of the curves is well reproduced, including the penetration depth of about $4 \mathrm{~nm}$ for the superconducting pairing wave function in $\mathrm{Cr}$. This remarkably small penetration depth in $\mathrm{Cr}$ is thus clearly proven to result from strong inelastic pair breaking scattering leading to an exponential damping of the pair wave function amplitude within the Cr-layer.

There is, however, an additional interesting experimental detail in Fig. 10 which the applied model fails to describe even in qualitative terms. This is the drop of $T_{c}\left(d_{C r}\right)$ for $d_{C r} \geq 4 \mathrm{~nm}$, clearly seen in Fig. 10. This feature was attributed to a transition of the entire Cr-layer from a non-magnetic state to an incommensurate SDW state at $d_{C r} \sim 4 \mathrm{~nm}$. The assumption of a strong suppression of the Cooper pair density by the transition of the Cr layer from a non-magnetic to a spin density wave state is plausible by the following reason. BCS-ordering and SDW-ordering in the same region of the Fermi surface can be considered as competing electronic ordering phenomena. In a theoretical paper on studying this problem (see, e.g., [78]), it was shown that those parts of the Fermi surface where the nesting feature leads to a SDW state the formation of the BCS-gap is suppressed and the superconducting transition temperature is reduced.

The study of the superconducting proximity effect in $\mathrm{Fe} / \mathrm{Cr} / \mathrm{V} / \mathrm{Cr} / \mathrm{Fe}$ gave new results concerning the magnetic phase transition in the Cr-layer, demonstrated a strong screening of the ferromagnetic exchange field of Fe by the interleaved Cr-layers and allowed to estimate the upper limit of the thickness of the screening Cr-layers for a spin valve to operate.

A novel approach for a realization of the superconducting spin valve design originally proposed by Sungjun et al [73] was also undertaken recently. The idea [79] for the realization of such a device was to choose as the non-magnetic interlayer $\mathrm{N}$ in the $\mathrm{S} / \mathrm{F} 1 / \mathrm{N} / \mathrm{F} 2 /$ layer scheme an interlayer with a thickness corresponding exactly to an antiferromagnetic interlayer exchange coupling between F1 and F2 [80]. Then, one can rotate the relative magnetization direction of F1 and F2 from antiparallel to parallel in an external field and observe the accompanying shift of the $T_{c}$.

The experimental system of choice was the epitaxial superlattice system $\mathrm{MgO}(100) /\left[\mathrm{Fe}_{2} \mathrm{~V}_{11}\right]_{20} / \mathrm{V}\left(d_{V}\right)$. (The index denotes the number of monolayers.) There are several reasons that make the choice of the epitaxial $(\mathrm{V} / \mathrm{Fe})$-system favorable for demonstrating the superconducting spin valve effect: First, it is the superior quality of the $\mathrm{Fe} / \mathrm{V}$ interface in the superlattice $[81,82,83,84]$ that guarantees a high interface transparency and weak diffusive pair breaking scattering at the interface. Second, the $\mathrm{Fe}_{2}$ layers have a thickness $d_{F}$ of about $0.3 \mathrm{~nm}$ only, whereas for the decay length of the superconducting pair density $\xi_{F} \sim 0.7 \mathrm{~nm}$ holds (see, e.g., [61]). Thus the pair wave function within the $\mathrm{Fe}_{2}$-layer will only be weakly damped and the condition $d_{F} / \xi_{F}<0.5$ optimal for observing the superconducting spin valve effect will be fulfilled [73]. In Fig. 12 we reproduce the magnetization curve of a $\left[\mathrm{Fe}_{2} \mathrm{~V}_{11}\right]_{20}$ - superlattice measured at $10 \mathrm{~K}$. The shape of the hysteresis shows that the interlayer exchange coupling is antiferromagnetic with a ferromagnetic saturation field of 


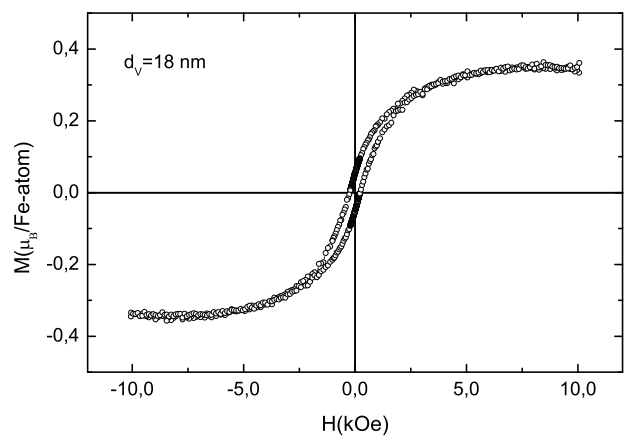

Fig. 12. Magnetization hysteresis loop of the sample $\left[\mathrm{Fe}_{2} \mathrm{~V}_{11}\right]_{20} / \mathrm{V}(18 \mathrm{~nm})$ measured at $10 \mathrm{~K}$.

$H_{\text {sat }}=6 \mathrm{kOe}$. The upper critical magnetic field for the field direction parallel and perpendicular to the film plane is plotted in Fig. 13 for several samples. For a two dimensional (2D) thin film with the magnetic field perpendicular or

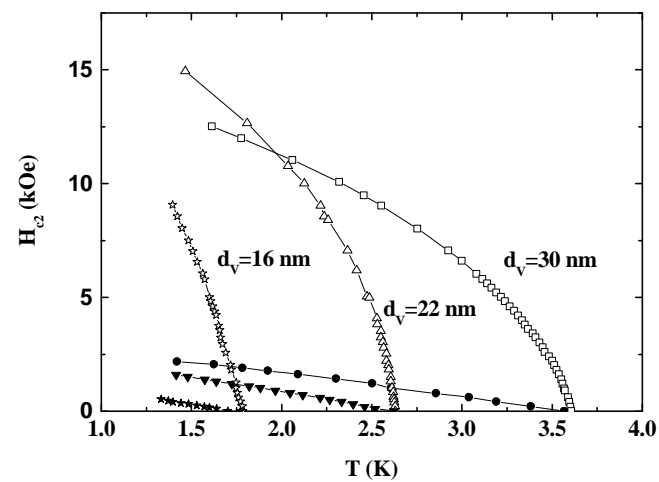

Fig. 13. Upper critical magnetic field versus temperature with the field applied parallel and perpendicular to the film plane for three samples $\left[\mathrm{Fe}_{2} \mathrm{~V}_{11}\right]_{20} / \mathrm{V}\left(d_{V}\right)$. The thickness $d_{V}$ is given in the figure, the open symbols refer to the magnetic field direction parallel to the film plane, the solid symbols refer to the direction perpendicular to the plane.

parallel to the film plane the classic result for the upper critical field is [85]:

$$
\begin{gathered}
H_{c 2}^{\text {perp }}=\frac{\Phi_{0}}{2 \pi \xi^{2}(0)}\left(1-\frac{T}{T_{c}}\right) \\
H_{c 2}^{p a r}=\frac{\Phi_{0}}{2 \pi \xi(0)} \frac{\sqrt{12}}{d_{s}} \sqrt{\left(1-\frac{T}{T_{c}}\right)}
\end{gathered}
$$


with the flux quantum $\Phi_{0}$, the thickness of the film $d_{S}$ and the GinzburgLandau correlation length $\xi(0)$ related to Pippard's correlation length $\xi_{s}$ as $\xi(0)=1.6 \xi_{s}$.

The measurements of the upper critical field for $\mathrm{Fe} / \mathrm{V} / \mathrm{Fe}$ trilayers for parallel orientation of the magnetic field relative to the film plane is perfectly described by Eq. (16), as it was observed earlier [86, 87]. In Figs. 14a and 14b
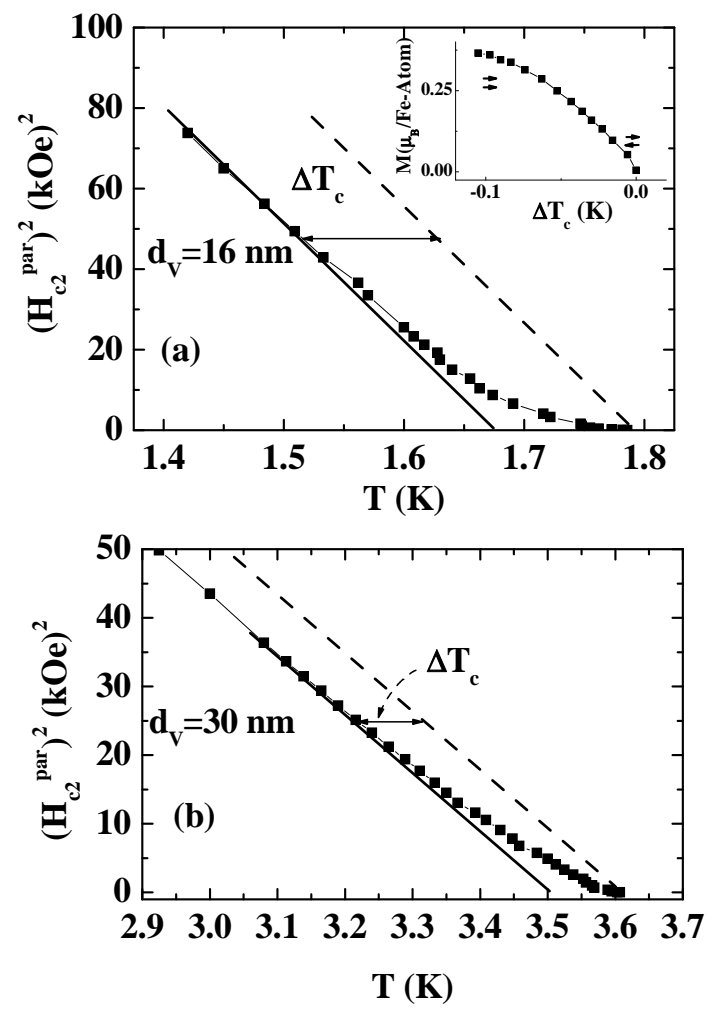

Fig. 14. Square of the parallel upper critical magnetic field versus temperature for the sample $\left[\mathrm{Fe}_{2} / \mathrm{V}_{11}\right]_{20} / \mathrm{V}(16 \mathrm{~nm})$ (a) and $\left[\mathrm{Fe}_{2} / \mathrm{V}_{11}\right]_{20} / \mathrm{V}(30 \mathrm{~nm})$ (b). The full straight line is the extrapolation of the linear temperature dependence for higher fields, the dashed line is the theoretical curve expected if the magnetization of the superlattice would not change. $\Delta T_{c}$ is the shift of the superconducting transition temperature between the superlattice in the antiferromagnetic state and in ferromagnetic saturation. The inset in panel (a) depicts the shift of the superconducting transition temperature with the magnetization of the $\left[\mathrm{Fe}_{2} / \mathrm{V}_{11}\right]_{20}$ superlattice

the square of parallel upper critical field are plotted together with the straight line that describes the temperature dependence for fields above $6 \mathrm{kOe}$ perfectly. Below $H=6 \mathrm{kOe}$ there is an increasing deviation from the straight line. From the extrapolation of the straight line one gets a superconducting 
transition temperature $T_{c}$ that is more than $0.1 \mathrm{~K}$ below the true transition temperature measured at zero field. A comparison with the magnetization curve in Fig. 12 shows that the ferromagnetic saturation field of $6 \mathrm{kOe}$ is correlated with the first deviation of $H_{c 2}^{2}(T)$ from the straight line in Fig. 14a and 14b. From this one can infer that the deviation of the upper critical field from the 2D-behavior in Fig. 14 is caused by the gradual change of the sublattice magnetization direction of the $\left[\mathrm{Fe}_{2} / \mathrm{V}_{11}\right]_{20}$-superlattice from parallel above $6 \mathrm{kOe}$ to antiparallel in zero field. For the sample with $d_{V}=16 \mathrm{~nm}$ in Fig. 14a $T_{c}=1.78 \mathrm{~K}$ in the antiferromagnetic state, while in the ferromagnetic saturation we extrapolate $T_{c}=1.67 \mathrm{~K}$. The temperature difference $\Delta T_{c}=0.11 \mathrm{~K}$ is the anticipated superconducting spin valve effect.

These experiments clearly demonstrate that the superconducting transition temperature of the $\mathrm{V}$-film reacts sensitively on the mutual magnetization orientation of the $\mathrm{Fe}_{2}$ layers of an antiferromagnetically coupled $\left[\mathrm{Fe}_{2} \mathrm{~V}_{11}\right]_{20}$ superlattice. Actually the ferromagnetic layers in this system cannot be switched from the parallel to the antiparallel state, since the parallel state needs the application of a strong external magnetic field. At the same time, it should be in principle possible to construct a switching device by replacing the antiferromagnetically coupled $[\mathrm{Fe} / \mathrm{V}]$ superlattice by a conventional spin valve trilayer system.

\subsection{Odd triplet superconductivity in $\mathrm{S} / \mathrm{F} / \mathrm{S}$ structures}

In section 2.3 a theoretical model predicting a possible robust triplet proximity effect in S/F structures has been described. The mechanism is operational in the presence of a rotating magnetization at the S/F interface. Recently Sosnin et al [13] presented the first clear experimental indication of this type of proximity effect using an Andreev type of reflectometer and an S/F/S mesoscopic thin film structure. The design of the reflectometer is depicted schematically in Fig. 15a. It consists of a superconducting Al-loop with an area of $20 \mu \mathrm{m}^{2}$ with a narrow gap bridged by a ferromagnetic Ho-stripe. The distance between the two $\mathrm{Al} / \mathrm{Ho}$ contact points was more than one order of magnitude larger than the singlet magnetic coherence length $\xi_{F 0}$. A rotating magnetization at the $\mathrm{S} / \mathrm{F}$ interface is established here by the intrinsic conical ferromagnetism of Ho (see Fig. 15 b). The essential experimental finding is that below the critical temperature $T_{c}$ of $\mathrm{Al}$ the resistance of the Ho wire exhibits oscillations as a function of the superconducting phase difference between the two interfaces of the Ho-stripe with the superconducting $\mathrm{Al}$ ring, as shown in Fig. 16a. The phase difference was generated by varying the magnetic flux penetrating the Al-loop. The period of the oscillations corresponds to the flux quantum $\Phi_{0}=2 \cdot 10^{-7} \mathrm{Gcm}^{2}$ and gives rise to the sharp peaks in the Fourier spectrum of oscillations (Fig. 16b). Estimates show that for the relative amplitude of the conductance oscillations $\Delta R / R_{F} \simeq 10^{-4}$ ( $R_{F}$ is the resistivity of the ferromagnetic wires) is expected. These oscillations were observed for the samples with a distance between the $\mathrm{Al} / \mathrm{Ho}$ contact points interfaces of up to 
(a)

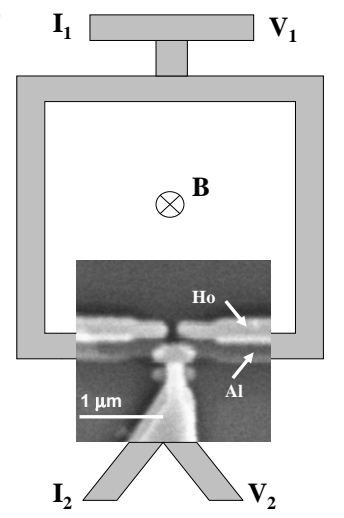

(b)

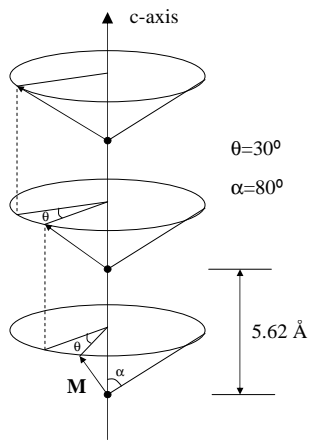

Fig. 15. a) Experimental set-up and SEM micrograph of S/F/S junction area prepared by shadow evaporation. b) Magnetic structure of Ho: magnetization $\mathbf{M}$ rotates by $30^{\circ}$ each atomic layer along $c$-axis at an angle of $80^{\circ}$ to this axis.

$L_{F}=160 \mathrm{~nm}$. Such a long-range phase coherence cannot be explained by the proximity effect involving the penetration of the ordinary singlet pairs, since the upper limit for the singlet penetration depth $\xi_{F 0}$ is equal to the electron mean free path $l$ which for was $l \approx 6 \mathrm{~nm}$. Thus, the observed oscillations of the magnetoresistance seem to originate from the long-range penetration of a helical triplet component of superconductivity generated in a ferromagnetic conductor and induced by the presence of a rotating magnetization.

Recently Keizer et al [15] studied lateral S/F/S Josephson junctions combining the strong ferromagnet $\mathrm{CrO}_{2}$ that belongs to the group of half-metals with full spin polarization of the electrons at the Fermi level and the conventional s-wave superconductor NbTi. They observed a Josephson supercurrent prevailing over very long length scales up to $\sim 1 \mu \mathrm{m}$. This is by orders of magnitude larger than expected for singlet correlations, which is of the order of $1 \mathrm{~nm}$. In addition to the long-range penetration of the superconducting pair density into $\mathrm{CrO}_{2}$, they found that the supercurrent strongly depended on the magnetization direction in the ferromagnet. On the basis of these findings Keizer et al attributed the long-range supercurrent to the triplet correlations. In this case of a half-metallic ferromagnet it is reasonable to assume that the LRTC is created at the S/F interface where spin-flip processes may happen [88].

Hints on the realization of the triplet proximity effect also came from recent magnetization data on hybrid structures consisting of multilayers of manganites $\left[\mathrm{La}_{0.33} \mathrm{Ca}_{0.67} \mathrm{MnO}_{3} / \mathrm{La}_{0.60} \mathrm{Ca}_{0.40 i} \mathrm{MnO}_{3}\right]_{15}$ in contact with a low$T_{c} \mathrm{Nb}$ superconductor [89]. 

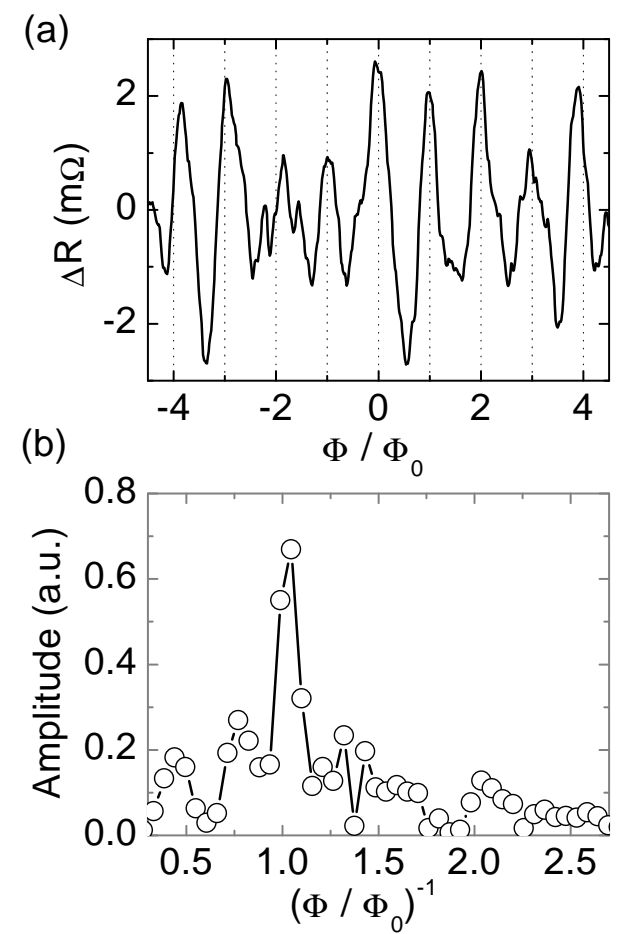

Fig. 16. a) Magnetoresistance oscillations of the sample shown in Fig. 15a measured at $\mathrm{T}=0.27 \mathrm{~K}$ as a function of normalized external flux through the loop. The sample resistance is $94.3 \Omega$. b) Fourier spectrum of the oscillations confirming the $h c / 2 e$ periodicity.

\subsection{Other proximity effects}

It seems natural and actually it is theoretically well established that the penetration of superconductivity from the S- into the F-layers is not the only possible proximity effect in $\mathrm{S} / \mathrm{F}$ systems (see section $2.5,2.6$ ). The proximity effect can also work in the reverse direction, i.e. the ferromagnetism from the F-layer can leak into the S-layer (inverse proximity effect) or the S-layer can modify the ferromagnetic state of the F-layer (cryptoferromagnetism). However, these effects are more subtle from the experimental point of view and are sill less established.

\section{Cryptoferromagnetism in $\mathrm{S} / \mathrm{F}$ layers}

As shown in Sec. 2.5, under certain conditions the ferromagnetic order in F-layers may be reconstructed by the action of the S-layer into a new magnetic domain state [17] or a cryptoferromagnetic state [18]. The basic physical reason for this behavior is that the destructive influence of the ferromagnetic 
exchange field on the superconductivity can be considerably reduced if the ferromagnetic state is modified in such a manner that the exchange field cancels when averaged over the superconducting coherence length.

The first hint in favor for a reconstruction of the ferromagnetic state below the superconducting transition temperature was obtained from the anomalous temperature dependence of the ferromagnetic resonance (FMR) line width observed in epitaxial $\mathrm{Fe} / \mathrm{Nb}$ bilayers below $T_{c}$ [48]. However, a quantitative estimate using the theory of Buzdin and Bulaevskii [17] rase doubts in this interpretation, since the effect in $\mathrm{Fe} / \mathrm{Nb}$ should only occur at an Fe-layer thickness an order of magnitude smaller than observed experimentally. Later Bergeret et al [18] studied theoretically the possibility of a non-homogeneous magnetic order of a ferromagnetic film placed on top of a bulk superconductor. They also concluded that due to the large magnetic stiffness constant in $\mathrm{Fe}$, the cryptoferromagnetic state can hardly be realized using pure Fe films. These considerations suggested that the tendency to a reconstruction of the ferromagnetic state observed experimentally in $\mathrm{Fe} / \mathrm{Nb}$ might be caused by a granular structure of the very thin Fe layers.

Quantitative estimates by Bergeret et al [18] showed that the transition from the ferromagnetic to the cryptoferromagnetic state should be observable in a ferromagnet with a magnetic stiffness constant an order of magnitude smaller than that of pure Fe. This can be achieved by dilution of Fe in suitable alloy systems, a favorable choice being $\mathrm{Pd}_{1-x} \mathrm{Fe}_{x}$ at small $\mathrm{x}$ due to its low and tunable Curie temperature.

In an FMR study for a series of samples $\mathrm{V} / \mathrm{Pd}_{1-x} \mathrm{Fe}_{x}$ the temperature dependence of the effective magnetization $4 \pi M_{\text {eff }}=4 \pi M-\left(2 K_{u} / M\right)(M$ is the saturation moment of the ferromagnet and $K_{u}$ is the perpendicular anisotropy constant) was measured [49]. The low-temperature part of $4 \pi M_{e f f}(T)$ is depicted in Fig. 17. One observes a decrease of the effective magnetization

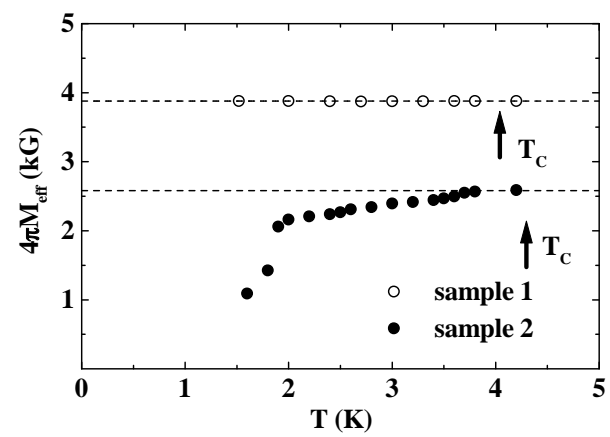

Fig. 17. Low-temperature parts of $4 \pi M_{e f f}(T)$ for the sample 1 with $d_{V}=37.2 \mathrm{~nm}$, $d_{P d-F e}=4.4 \mathrm{~nm}, T_{c}=4.0 \mathrm{~K}$ and sample 2 with $d_{V}=40 \mathrm{~nm}, d_{P d-F e}=1.2 \mathrm{~nm}, T_{c}=4.2$ $\mathrm{K}$. The arrows show the $T_{c}$-values at the resonance field $H_{0}$. 
$4 \pi M_{\text {eff }}$ below $T_{c}$ for the sample 2 (Fig. 17) but not for sample 1. A decrease of $4 \pi M_{\text {eff }}$ can be caused by a decrease of the saturation magnetization $M$ or by an increase of the perpendicular uniaxial anisotropy constant $K_{u}$. A comparison of FMR results for films with different thickness of the ferromagnetic layers leads to the conclusion that $K_{u}$ is very small and the decrease of $4 \pi M_{\text {eff }}$ must be caused by a decrease of the saturation magnetization $M$. This suggests that the decrease of the saturation magnetization below $T_{c}$ is caused by a reconstruction of the ferromagnetic state.

An estimate following the phase diagram by Bergeret et al [18] (Fig. 5) gave the parameters $a \sim 1.2$ and $\lambda \sim 1.3 \cdot 10^{-3}$ for sample 2 with $d_{M} \sim 1.2$ $\mathrm{nm}$ and $T_{\text {Curie }} \sim 100 \mathrm{~K}$. In accordance with the phase diagram of Bergeret et al (Fig. 5) this implies that starting from $\tau \sim 0.2(T \sim 3.2 \mathrm{~K})$ a transition from the ferromagnetic to the cryptoferromagnetic state should take place, as it is actually observed experimentally. For the sample 1 with $d_{M} \sim 4.4$ $\mathrm{nm}$ and $T_{\text {Curie }} \sim 250 \mathrm{~K}$ we have $a \sim 20$ and $\lambda \sim 1.4 \cdot 10^{-2}$. With these parameter values the ferromagnetic state should be stable at any temperature, in agreement with the experimental result.

Thus, these estimates support the conclusion that a phase transition from the ferromagnetic state to the cryptoferromagnetic state occurs in sample 2. However, one cannot completely exclude that the anomalous temperature dependence of $M_{\text {eff }}$ might be due to the screening of the magnetic moments of the ferromagnetic layer by the polarized Cooper pairs, as discussed in Sec. 2.6 and in the next section.

\section{Inverse proximity effect}

Up to now any unequivocal experimental evidence for the penetration of the magnetization from the ferromagnetic side into the superconducting side of an S/F bilayer, as discussed in Sec. 2.6, does not exist. First interpretations in this direction have been published only recently [89, 90]. Stahn et al [90] studied the magnetization profile of $\left[\mathrm{YBa}_{2} \mathrm{Cu}_{3} \mathrm{O}_{7} / \mathrm{La}_{2 / 3} \mathrm{Ca}_{1 / 3} \mathrm{MnO}_{3}\right]$ multilayers using neutron reflectometry. From a change of the reflectivity curves below the superconducting transition temperature they could propose two possible magnetization profiles. In the first one a magnetic moment within the superconducting layer antiparallel to that of the F-layer exists, which is consistent with the inverse proximity effect. The second profile had a magnetically "dead" interlayer region in the F-layer with zero magnetic moment. Stahn et al [90] argue in favor of the first possibility but the situation is not yet settled.

Stamopoulos et al [89] presented magnetization measurements on multilayers of manganites $\left[\mathrm{La}_{0.33} \mathrm{Ca}_{0.67} \mathrm{MnO}_{3} / \mathrm{La}_{0.60} \mathrm{Ca}_{0.40} \mathrm{MnO}_{3}\right]_{15}$ in contact with a low- $T_{c}$ superconductor. They came to the conclusion that the superconductor below $T_{c}$ becomes ferromagnetically coupled to the multilayer. Since it is expected that for the singlet pairing the magnetization of $\mathrm{F}$ penetrates into $\mathrm{S}$ antiferromagnetically, the authors conclude that a spin-triplet supercon- 
ducting component forms and penetrates into the F-layer thus inducing the ferromagnetic coupling observed experimentally.

\section{Summary and Conclusions}

The main purpose of the present paper was to review the status of the research on the proximity effects in the $\mathrm{S} / \mathrm{F}$ layer systems from the experimental as well as from the theoretical point of view.

Peculiarities of the $\mathrm{S} / \mathrm{F}$ proximity effect originating from the penetration of the condensate function into the ferromagnet that have been discussed controversially in the beginning seem to be well established by now. The $\mathrm{S} / \mathrm{F} / \mathrm{S}$ Josephson junctions with the $\pi$-coupling are, e.g., even suggested as basic units for realization of Q-bits for quantum computing [91].

It has become already traditional in the field of the $\mathrm{S} / \mathrm{F}$ proximity effect that theory is somewhat ahead of experiment. The situation persists and intriguing theoretical predictions are still waiting for the first experimental verifications or further experimental support. One of these predictions concerns the unconventional superconductivity in $\mathrm{S} / \mathrm{F}$ systems. The experimental realization is difficult, since the unconventional superconductivity expected here, namely odd triplet superconductivity, can only be generated by a rotating magnetization at the interface. Nevertheless, the first experimental indications of its existence have already been reported. What is important, the odd triplet superconductivity is insensitive to scattering on non-magnetic impurities and this is certainly helpful for an experimental observation.

We should also mention further recent ideas on how to identify the oddtriplet superconductivity [92, 93, 94].

The inverse proximity effect, i.e. the penetration of the magnetic order parameter into a superconductor, has not been clearly observed experimentally until now. However, indications on the closely related effect, namely the decreasing of the total ferromagnetic moment below $T_{c}$, already exist. Yet, it is not easy to clarify to what extent the non-homogeneous distribution of $M_{F}$ produced in the ferromagnet below $T_{c}$ contributes to the effect. The best way to observe the spin screening of $M_{F}$ is either probing directly the spatial distribution of the magnetic field using neutron scattering or by measuring muon spin resonance. Since the magnetic moment $M$ varies on the macroscopic length $\xi_{S}$, it should be possible to detect it.

A considerable work still remains to be done on the experimental side. Only careful material selection, optimization of film preparation and device design will enable one clarification of all the complex phenomena that may occur in the $\mathrm{S} / \mathrm{F}$ proximity systems. Very promising seems the study of $\mathrm{F} / \mathrm{S}$ structures with comparable ferromagnetic Curie and superconducting transition temperatures. Combining elemental superconductors with elemental ferromagnets, as was done in the majority of papers on the $\mathrm{S} / \mathrm{F}$ proximity effect 
published until now, is not the best way for the observation of the proximity effects because the ferromagnetic exchange energy is orders of magnitude larger than the superconducting condensation energy. In this case the ferromagnetic state can hardly be modified by the superconductor. Rare earth based ferromagnetic compounds with low Curie temperatures would, in principle, be a better choice. Combining high- $T_{c}$ superconductors with ferromagnetic oxides is another promising option.

The authors are grateful for the support by the Deutsche Forschungsgemeinschaft (DFG) within SFB 491. One of us (AFV) would like to acknowledge financial support from DFG within the project Mercator-Gastprofessoren.

\section{References}

1. P. G. de Gennes, Superconductivity of Metals and Alloys (Benjamin, New York, 1966)

2. G. Deutscher, Rev. Mod. Phys. 77, 109 (2005)

3. L.N. Bulaevskii, V.V. Kuzii, and A.A. Sobyanin, Pis'ma Zh. Eksp. Teor. Fiz. 25, 314 (1977) (JETP 25, 290 (1977)

4. V.V. Ryazanov, V.A. Oboznov, A. Yu. Rusanov, A.V. Veretennikov, A.A. Golubov, and J. Aarts, Phys. Rev. Lett. 86, 2427 (2001)

5. Y. Blum, A. Tsukernik, M. Karpovski, and A. Palevski, Phys. Rev. Lett 89 $187004(2002)$

6. L. R. Tagirov, Phys. Rev. Lett. 83, 2058 (1999)

7. A.I. Buzdin, A.V. Vedyaev, and N.V. Ryzhanova, Europhys. Lett. 48, 686 (1999)

8. J.R. Schrieffer, Theory of Superconductivity, Benjamin (1964)

9. C.C. Tsuei and J.R. Kirtley, Rev. Mod. Phys. 72, 969 (2000)

10. V.P. Mineev and K.V. Samokhin, Introduction to Unconventional Superconductivity (Gordon and Breach, Amsterdam, 1999)

11. A.P. Mackenzie and Y. Maeno, Rev. Mod. Phys. 75, 657 (2003)

12. I. Eremin, D. Manske, S.G. Ovchinnikov, and J.F. Annett, Ann. Phys. 13, 149 (2004)

13. I. Sosnin, H. Cho, V.T. Petrashov, and A.F. Volkov, Phys. Rev. Lett. 96, 157002 (2006)

14. V. Pena, Z. Sefrioui, D. Arias, C. Leon, J. Santamaria, J.L. Martinez, S.G.E. te Velthuis, and A. Hoffman: Phys. Rev. Lett. 94, 057002 (2005)

15. R.S. Keizer, S.T.B. Goennenwein, T.M. Klapwijk, G. Miao, G. Xiao, and A. Gupta, Nature 439, 825 (2006)

16. P.W. Anderson and H. Suhl, Phys. Rev. 116, 898 (1959)

17. A.I. Buzdin, L.N. Bulaevskii, Sov. Phys. JETP 67, 576 (1988)

18. F.S. Bergeret, K.B. Efetov, A.I. Larkin, Phys. Rev. B 62, 11872 (2000)

19. F.S. Bergeret, A.F. Volkov and K.B. Efetov, Phys. Rev. B 69, 174504 (2004); Europhys. Lett. 66, 111 (2004)

20. C.L. Chien, D. Reich, J. Magn. Magn. Mater. 200, 83 (1999)

21. I.A. Garifullin, J. Magn. Magn. Mater. 240, 574 (2002) 
22. Yu.A. Izyumov, Yu.N. Proshin, and M.G. Khusainov, Uspehi Fiz. Nauk 172, 113 (2002) [ Phys. Usp. 45, 109 (2002)]

23. A.A. Golubov, M.Yu. Kupriyanov, and Il'ichev, Rev. Mod. Phys. 76,411 (2004)

24. A.I. Buzdin, Rev. Mod. Phys. 77, 935 (2005)

25. F.S. Bergeret, A.F. Volkov, K.B. Efetov, Rev. Mod. Phys. 77, 1321 (2005)

26. A.J. Leggett, Rev. Mod. Phys. 47, 331 (1975)

27. D. Vollhardt and P. Wölfle, The superfluid phases of He 3 (Taylor and Francis, London, New York, Philadelphia) (1990)

28. Z. Radović, M. Ledvij, L. Dobrosavljević-Grujic, A.I. Buzdin, and J.R. Clem, Phys. Rev. B 44, 759 (1991)

29. A.V. Demler, G.B. Arnold and M.R. Beasley, Phys. Rev. B 55, 15174 (1997)

30. L.R. Tagirov, Physica C 307, 145 (1998)

31. I. Baladie and A.I. Buzdin, Phys. Rev B 67, 014523 (2003)

32. A. Bagrets, C. Lacroix, and A. Vedyaev, Phys. Rev. B 68, 054532 (2003)

33. Y.V. Fominov, N.M. Chtchelkatchev, and A.A. Golubov, Phys. Rev. B 66, 014507 (2002)

34. I. Baladie and A.I. Buzdin, Phys. Rev. B 64, 224514 (2001)

35. F.S. Bergeret, A.F. Volkov, and K.B. Efetov, Phys. Rev. B 66, 184403 (2002)

36. F.S. Bergeret, A.F. Volkov and K.B. Efetov, Phys. Rev. Lett. 86, 3140 (2001)

37. G. Sarma, J. Phys. Chem. Solids 24, 1029 (1963)

38. A.I. Buzdin, L.N. Bulaevskii and S.V. Panyukov: Pis'ma Zh. Eksp. Teor. Fiz. 35, 147 (1982) [JETP Lett. 35, 178 (1982)]

39. T. Kontos, M. Aprili, J. Lesueur, F. Genet, B. Stephanidis, and R. Boursier, Phys. Rev. Lett. 89, 137007 (2002)

40. A. Bauer, J. Bentner, M. Aprili, M. L. D. Rocca, M. Reinwald, W. Wegscheider, and C. Strunk, Phys. Rev. Lett. 92, 217001 (2004)

41. V. L. Berezinskii, Pis'ma Zh. Eksp. Teor. Fiz. 20, 628 (1974) [JETP Lett. 20, $287(1974)]$

42. F.S. Bergeret, A.F. Volkov and K.B. Efetov, Phys. Rev. Lett. 86, 4096 (2001)

43. A.F. Volkov, F.S. Bergeret, and K.B. Efetov: Phys. Rev. Lett. 90, 117006 (2003); Phys. Rev. B 68, 064513 (2003)

44. A. Kadigrobov, R.I. Shekhter and M. Jonson, Europhys. Lett. 54, 394 (2001)

45. A. F. Volkov, A. Anishchanka, and K. B. Efetov, Phys. Rev. B 73, 104412 (2006)

46. T. Champel and M. Eschrig, Phys. Rev. B 72, 054523 (2005).

47. A.F. Volkov, Ya.V. Fominov, and K.B. Efetov, Phys. Rev. B 72, 184504 (2005)

48. Th. Mühge, N.N. Garif'yanov, Yu.V. Goryunov, K. Theis-Bröhl, K. Westerholt, I.A. Garifullin, and H. Zabel: Physica C 296, 325 (1998)

49. I.A. Garifullin, D.A. Tikhonov, N.N. Garif'yanov, M.Z. Fattakhov, K. TheisBröhl, K. Westerholt, and H. Zabel, Appl. Magn. Reson. 22, 439 (2002)

50. M.Yu. Kharitonov, A.F. Volkov, and K.B. Efetov, Phys. Rev. B 73, 054511 (2006)

51. T. Löfwander, T. Champel, J. Durst, and M. Eschrig, Phys. Rev. Lett. 95, $187003(2005)$

52. Th. Mühge, N.N. Garif'yanov, Yu.V. Goryunov, G.G. Khaliullin, L.R. Tagirov, K. Westerholt, I.A. Garifullin, and H. Zabel, Phys. Rev. Lett. 77, 1857 (1996)

53. Th. Mühge, K. Westerholt, H. Zabel, N.N. Garif'yanov, Yu.V. Goryunov, I.A. Garifullin, G.G. Khaliullin: Phys. Rev. B 55, 8945 (1997)

54. J.S. Jiang, D. Davidović, D.H. Reich, and C.L. Chien, Phys. Rev. Lett. 74, 314 (1995) 
55. J.S. Jiang, D. Davidović, D.H. Reich, and C.L. Chien, Phys. Rev. B 54, 6119 (1996)

56. C.L. Chien, J.S. Jiang, J.Q. Xiao, D. Davidović, and D.H. Reich, J. Appl. Phys. 81, 5358 (1997)

57. F.Y. Ogrin, S.L. Lee, A.D. Hillier, A. Mitchell, and T.-H. Shen, Phys. Rev. B 62, $6021(2000)$

58. W. Guichard, M. Aprili, O. Bourgeois, T. Kontos, J. Lesueur, and P. Gandit, Phys. Rev. Lett. 90, 167001 (2003)

59. M.G. Khusainov and Yu.N. Proshin, Phys. Rev. B 56, 14283 (1997); Erratum: Phys. Rev. B 626832 (2000)

60. J. Aarts, J.M.E. Geers, E. Brück, A.A. Golubov, and R. Coehoorn, Phys. Rev. 56, 2779 (1997)

61. L. Lazar, K. Westerholt, H. Zabel, L.R. Tagirov, Yu.V. Goryunov, N.N. Garif'yanov, and I.A. Garifullin, Phys. Rev. B 61, 3711 (2000)

62. I.A. Garifullin, D.A. Tikhonov, N. N. Garif'yanov, L. Lazar, Yu.V. Goryunov, S.Ya. Khlebnikov, L.R. Tagirov, K. Westerholt, and H. Zabel, Phys. Rev. B. 66, 020505(R) (2002)

63. A.B. Pippard, Rep. Prog. Phys. 23, 176 (1960)

64. V. Zdravkov, A. Sidorenko, G. Obermeier, S. Gsell, M. Schreck, C. Müller, S. Horn, R. Tidecks, L.R. Tagirov, Phys. Rev. Lett. 97, 057004 (2006)

65. G. Jacob, V.V. Moshchalkov, and Bruynseraede, Appl. Phys. Lett. 66, 2564 (1995)

66. N.-C. Yeh, R.P. Vasquez, C. C. Fu, A.V. Samoilov, Y. Li, and K. Vakili, Phys. Rev. B 60, 10522 (1999)

67. Z. Sefrioui, D. Arias, V. Pena, J.E. Villegas, M. Varela, P. Prieto, C. Leon, J.L. Martinec, and J. Santamaria, Phys. Rev. B 67, 214511 (2003)

68. Todd Holden, H.-U. Habermeier, G. Gristiani, A. Golnik, A. Boris, A. Pimenov, J. Humlicek, O.I. Lebedev, G. Van Tendeloo, B. Keimer, and C. Bernhard, Phys. Rev. B 69, 064505 (2004)

69. S. Soltan, J. Albrecht, and H.-U. Habermeier, Phys. Rev. B 70, 144517 (2004)

70. J.Y. Gu, C.-Y. You, J.S. Jiang, J. Pearson, Ya.B. Bazaliy, and S.D. Bader, Phys. Rev. Lett. 89, 267001 (2002)

71. A. Potenza and C.H. Marrows, Phys. Rev. B 71, 180503 (2005)

72. A.Yu. Rusanov, M. Hesselberth, J. Aarts, and A.I. Buzdin, Phys. Rev. Lett. 93, $057002(2004)$

73. Sangjun Oh, D. Youm, and M.R. Beasley, Appl. Phys. Lett. 71, 2376 (1997)

74. I. A. Garifullin, D. A. Tikhonov, N. N. Garif'yanov, M. Z. Fattakhov, L.R. Tagirov, K. Theis-Bröhl, K. Westerholt, and H. Zabel, Phys. Rev. B 70, 054505, (2004)

75. M. Hübener, D.A. Tikhonov, I.A. Garifullin, K. Westerholt, and H. Zabel, J. Phys.: Condens. Matter 14, 8687 (2002)

76. A.A. Abrikosov and L.P. Gor'kov, Zh. Eksp. Teor. Fiz. 39, 1781 (1960) [Sov. Phys. JETP 12, 1243 (1961)]

77. B.P. Vodopyanov, L.R. Tagirov, H.Z. Durusoy and A.V. Berezhnov, Physica C 366, 31 (2001)

78. K. Machida, J. Phys. Soc. Jpn. 50, 2195 (1981)

79. K. Westerholt, D. Sprungmann, H. Zabel, R. Brucas, B. Hjörvarsson, D.A. Tikhonov, and I.A. Garifullin, Phys. Rev. Lett. 95, 097003 (2005)

80. P. Bruno and C. Chappert, Phys. Rev. B 46, 261 (1992) 
81. B. Hjörvarsson, J.A. Dura, P. Isberg, T.U. Watanabe, T. J. Udovic, G. Andersson, and C.F. Majkrzak, Phys. Rev. Lett. 79, 901 (1997)

82. G. Andersson, B. Hjörvarsson, and H. Zabel, Phys. Rev. B 55, 15905 (1997)

83. P. Isberg, B. Hjörvarsson, R. Wräppling, E. B. Svedvberg, and L. Hultman, Vacuum 48, 483 (1997)

84. V. Uzdin, K. Westerholt, H. Zabel, and B. Hjörvarsson, Phys. Rev. B 68, 214407 (2003)

85. M. Tinkham, Introduction to Superconductivity (McGraw-Hill, New York, 1978) pp. 130-131

86. H.J. Wong, B.Y. Jin, H.Q. Yang, J.B. Ketterson, and J.E. Hillard, J. Low Temp. Phys. 63, 307 (1986)

87. P. Koorevaar, Y. Suzuki, R. Coehoorn, and J. Aarts, Phys. Rev. B 49, 441 (1994)

88. M. Eschrig, J. Kopu, J. C. Cuevas, and G. Schön, Phys. Rev. Lett. 90, 137003 (2003)

89. D. Stamopoulos, N. Moutis, M. Pissas, and D. Niarchos, Phys. Rev. B 72, 212514 (2005)

90. J. Stahn, J. Chakhalian, Ch. Niedermeyer, J. Hoppler, T. Gutberlet, J. Voigt, F. Treubel, H-U. Habermeier, G. Cristiani, B. Keimer, and C. Bernhard, Phys. Rev. B 71, 140509 (2005) 70, 144517 (2004)

91. G. Blatter, V.B. Geshkenbein, and L.B. Ioffe, Phys. Rev. B. 63, 174511 (2001)

92. T. Yokoyama, Y. Tanaka, A.A. Golubov, cond-mat/0610608

93. Y. Tanaka, A.A. Golubov, S. Kashiwaya, cond-mat/0610017

94. V. Braude, Yu. V. Nazarov, cond-mat/0610037. 